\title{
DESAFÍOS ESTRATÉGICOS EN INSUFICIENCIA CARDÍACA AVANZADA. ASISTENCIA VENTRICULAR. ¿ESTÁ TODO DICHO?
}

\author{
STRATEGIC CHALLENGES IN ADVANCED HEART FAILURE. VENTRICULAR \\ ASSISTANCE. IS IT ALL SAID?
}

\section{JIMENA SANTILLÁN}

\section{RESUMEN}

La insuficiencia cardíaca avanzada es la etapa final de múltiples enfermedades cardiovasculares y constituye en sí misma una de las patologías más prevalentes y con mayor morbimortalidad a nivel mundial. Se sabe que el tratamiento médico óptimo ha demostrado mejorar la sobrevida en términos de progresión de enfermedad y muerte súbita, así como el cardiodesfibrilador implantable y la terapia de resincronización cardíaca.

A pesar de esto, un porcentaje no menor alcanza estadios avanzados en quienes el trasplante cardíaco puede ser una opción. Debido a las limitaciones actuales que esto implica, surgió el desarrollo de los dispositivos de asistencia ventricular (DAV). El propósito de esta monografía es revisar los aspectos clínicos y tecnológicos de los DAV. Se realizará un análisis de su evolución desde las primeras asistencias, se evaluarán indicaciones, el momento oportuno para su implante así como sus contraindicaciones. Posteriormente, se realizará una revisión de los aspectos a tener en cuenta por parte del médico tratante para la evaluación precolocación, considerando los scores de riesgo disponibles, los aspectos psicosociales y el costo/beneficio de su utilización, para así poder evaluar el impacto y la factibilidad de su aplicación en la práctica clínica diaria.

Palabras clave: corazón auxiliar, insuficiencia cardíaca, trasplante de corazón.

\section{ABSTRACT}

Advanced heart failure is the final stage of multiple cardiovascular diseases, constituting in itself one of the most prevalent pathologies and with the highest morbidity and mortality worldwide. It is known that optimal medical treatment has been shown to improve survival in terms of disease progression and sudden death, as well as the implantable cardioverter defibrillator and cardiac resynchronization therapy. Despite this, a non-minor percentage reaches advanced stages in whom heart transplantation may be an option. Due to the current limitations that this implies, the development of ventricular assist devices (VAD) arose.

The purpose of this monograph is to review the clinical and technological aspects of VADs. An analysis of their evolution will be carried out from the first devices, indications will be evaluated, the opportune time for its implantation, as well as its contraindications. Subsequently, a review will be made on the aspects to be taken into account by the attending physician for the pre-placement evaluation, taking into account the risk scores available, psychosocial aspects and their cost / benefit; in order to evaluate the impact and feasibility of its application in daily clinical practice.

Keywords: heart-assist devices, heart failure, heart transplantation.

REVISTA CONAREC 2018;33(147):289-303 | DOI:10.32407/RCON/2018147/0289-0303

\section{INTRODUCCIÓN}

La insuficiencia cardíaca avanzada (ICA) es la etapa final de múltiples enfermedades cardiovasculares (ECV), y conforma en sí misma una de las patologías más prevalentes y con mayor morbimortalidad a nivel mundial. Se define por la presencia de deterioro severo de la fracción de eyección del ventrículo izquierdo (FEVI), síntomas graves progresivos y/o persistentes a pesar de máximo tratamiento médico, de dispositivos y quirúrgico constituyendo el estadio D de la clasificación de la American Heart Association (AHA) (Figura 1, Tabla 1) ${ }^{1-4}$.

En la última década se ha observado un aumento significativo en relación a cambios epidemiológicos que incluyen el envejecimiento poblacional, con el concomitante incremento de las comorbilidades, el desarrollo de nuevos métodos diagnósticos y tratamientos que prolongan la sobrevida y predisponen a múltiples reinternaciones ${ }^{5}$. En Argentina, la tasa de mortalidad por ECV fue en el 2014 de 148 por cien mil habitan-

1. Residente de Cardiología. Hospital Alemán. CABA.

$\triangle$ Correspondencia: Dra. Jimena Santillán. Av. Pueyrredón 1640. C1118AAT CABA. Rep.Argentina.jimenasantillan75@gmail.com.

Los autores declaran no poseer conflictos de intereses.

Recibido: 11/11/2017| Aceptado: 12/1/2018 tes y la insuficiencia cardíaca (IC) fue la principal causa de muerte ${ }^{6}$, representando el 18\% del total en adultos mayores de 45 años $^{7}$.

A nivel mundial, la IC también lidera la causa de muerte: en los Estados Unidos (EE.UU.) la prevalencia excede los 5,8 millones y cada año se diagnostican más de 550.000 casos nuevo ${ }^{5}$ con una tasa de mortalidad anual de aproximadamente 280 mil pacientes, de los cuales 250 mil presentan IC severa ${ }^{8}$. Las cardiomiopatías y la enfermedad coronaria constituyen las principales causas subyacentes ${ }^{9}$.

En el estudio de Framingham se señaló que la incidencia de IC se duplica por cada década de vida, siendo el ascenso más pronunciado en la mujer que en el hombre ${ }^{10}$. Además, la prevalencia es de $1 \%$ para menores de 50 años, $5 \%$ entre los 50 y 70 años, con un aumento del 10\% por cada década a partir de los 70 años ${ }^{8}$.

Se sabe que el tratamiento médico óptimo, que incluye inhibidores de la enzima convertidora de la angiotensina (IECA), betabloqueantes (BB), inhibidores de la aldosterona, antagonistas del receptor de la angiotensina II (ARA) -los cuales han demostrado mejorar la sobrevida en términos de progresión de enfermedad y muerte súbita- actualmente incorpora dispositivos como el cardiodesfibrilador implantable (CDI) y la terapia de resincronización cardíaca (TRC) por haber demostrado similares beneficios. A pesar de estos avances, un porcentaje no menor alcanza estadios avanzados; en estos, las estrategias terapéuticas son complejas y en muchas ocasiones no accesibles ${ }^{1-4}$.

El primer trasplante cardíaco realizado en 1967 revolucionó el pronóstico de la ICA, aportando una nueva opción al paciente refractario. Sin embar- 
Tabla 1. Estadios de la insuficiencia cardíaca propuestos por ACCF/AHA y la Clasificación Funcional de la NYHA.

\section{Clase funcional de la NYHA}

\section{Estadios de la ACCF/AHA

\begin{abstract}
estructuralt.
\end{abstract} \\ Estadios de la ACCF/AHA \\ B Pacientes asintomáticos con cardiopatía estructuralt.

\author{
Síntomas actuales o pasados de ICC asociados con cardiopatía estructural
} subyacente.†$$
\text { C subyacente. }
$$ \\ Pacientes asintomáticos con cardiopatía estructuralt.}

Insuficiencia cardíaca avanzada: Cardiopatía estructural avanzada y síntomas

severos en reposo, a pesar de terapia médica máxima. Se requieren intervenciones especializadas, como el tratamiento inotrópico hospitalario, trasplante o dispositivos de asistencia circulatoria

I Sin limitaciones de la actividad física.
I Sin limitaciones en la actividad física.
II Asintomático en reposo. La actividad habitual provoca síntomas
de IC.

III Marcada limitación de la actividad física. Asintomático en reposo.

\begin{abstract}
CF NYHA: Clase funcional New York Heart Association. ACCF: American College of Cardiology Foundation. AHA: American Heart Association. *: Hipertensión arterial, diabetes mellitus, obesidad, enfermedad coronaria (se excluyen de esta definición los pacientes con antecedentes de infarto agudo de miocardio). t: Antecedentes de infarto agudo de miocardio, disfunción sistólica, disfunción diastólica, hipertrofia del ventrículo izquierdo (determinadas por ecocardiograma o electrocardiograma), dilatación del ventrículo izquierdo, patologías valvulares, trastornos de la motilidad regional. Modificado de: Yancy CW, Jessup M, Bozkurt B, Butler J, Casey DE Jr, Drazner MH et al. 2013 ACCF/AHA guideline for the management of heart failure: a report of the American College of Cardiology Foundation/American Heart Association Task Force on practice guidelines. Circulation 2013;128(16):240-327.
\end{abstract}

go, nos enfrentamos al obstáculo que implica la desproporción en la relación donantes/receptor con listas de espera que superan los 80 días, con una media en pacientes en lista de urgencia de hasta 293 días ${ }^{11,12}$

Dicha escasez estaría explicada por diferentes motivos según las poblaciones analizadas: las nuevas leyes de tránsito y el perfeccionamiento de la industria automovilística, junto con las mejoras en leyes de seguridad y trabajo, que han resultado en un descenso de las muertes relacionadas a accidentes. Por otro lado, el continuo envejecimiento de la población llevó no solo a que el número de donantes haya disminuido sino también a que, los órganos procurados sean provenientes de personas de mayor edad, lo que se asocia a peores desenlaces postrasplante ${ }^{11,12}$. Es posible concluir por ello que, debido a las largas listas de espera por la escasa procuración de órganos, el número creciente de donantes con medidas de sostén avanzadas, los tiempos de isquemia condicionados por la extensión territorial y la ausencia de programas de gestión de salud, se ha hecho indispensable la búsqueda de alternativas al tratamiento médico y al trasplante cardíaco. Surge ante esta problemática el desarrollo de los dispositivos de asistencia ventricular (DAV), en un principio de corto plazo y en la actualidad de larga duración.

El propósito de esta monografía es revisar los aspectos clínicos y tecnológicos de los DAV. Se realizará un análisis de su evolución desde las primeras asistencias, se evaluarán indicaciones, el momento oportuno para su implante así como sus contraindicaciones. Posteriormente se realizará una revisión de los aspectos a tener en cuenta por parte del médico tratante para la evaluación precolocación considerando los scores de riesgo disponibles, aspectos psicosociales y el costo/beneficio de su empleo, para así poder evaluar el impacto y la factibilidad de su aplicación en la práctica clínica diaria.

\section{MATERIALES Y MÉTODOS}

Para realizar la siguiente investigación bibliográfica se utilizaron artículos originales, revisiones, editoriales, guías y diferentes publicaciones buscadas a través de los portales de Internet de la base de datos de la Biblioteca Nacional de los Estados Unidos Medline (PubMed) y Embase, en la base de revisiones sistemáticas Cochrane y en las bases de las principales revistas internacionales de cardiología y de medicina interna de la actualidad, así como en registros epidemiológicos nacionales y exper- tos en el tema. Se utilizó una restricción de idioma a inglés y español y sin restricción en las fechas de publicación.

Para la búsqueda se utilizaron los siguientes términos aislados o en combinación: "epidemiology of heart failure," "epidemiology of heart transplant", "history of VAD" "ventricular assist devices bridge to transplantation", "VAD bridge to transplant", "LVAD bridge to candidacy", "LVAD destination therapy", "ventricular circulatory support "', advanced heart failure," "complications of VAD", "mortality of VADs," "congestive heart failure therapy", "depression in heart failure," "prognosis of patients on wait list", "prolong wait list in heart failure" "evaluation of psychosocial risk in LVAD", "LVAD risk," "INTERMACS" El material fue clasificado por fecha y relevancia. En primer término, se analizaron los trabajos de revisión que luego generaron nuevas búsquedas bibliográficas. En segunda instancia, se consideraron los artículos originales, guías y editoriales con el objetivo de realizar un análisis crítico y profundo del tema.

\section{INSUFICIENCIA CARDÍACA AVANZADA Y EL DESARROLLO DE LAS ASISTENCIAS VENTRICULARES}

Conceptualmente la asistencia ventricular incluye a diversos dispositivos capaces de generar flujo circulatorio para sustituir parcial o totalmente la función del corazón en situaciones de falla aguda o crónica severas que no responden a otros tratamientos ${ }^{12}$.

En estudios recientes la disfunción sistólica se encuentra presente en el 5,5\% de las IC de la población anciana. En Estados Unidos (EE.UU.) la prevalencia de la IC excede los 5,8 millones, y de cada 550.000 casos diagnosticados por año cerca del $5 \%$ constituyen el estadio D de la $\mathrm{AHA}^{4}$ Por lo mencionado previamente acerca de la desproporción donante/ receptor, surge como alternativa la necesidad del implante de soporte circulatorio temporal hasta disponer del órgano apropiado o incluso en algunos casos como terapia final en aquellos que no son candidatos a trasplantarse $\mathrm{e}^{13-15}$.

En la década de 1950, con el advenimiento de las técnicas modernas de cirugía cardíaca, John Gibbon introdujo el uso clínico del bypass cardiopulmonar. De este modo se hizo posible realizar procedimientos de mayor complejidad y se establecieron los cimientos para el desarrollo de las asistencias ventriculares, llevando poco después a la introducción del 
soporte circulatorio fuera del quirófano. Los primeros en implantar un DAV para el tratamiento del shock cardiogénico fueron Cooley en 1960 y luego Liotta en 1963 $3^{16,17}$.

En 1964 el National Institutes of Health de EE.UU. desarrolló el Artificial Heart Program ${ }^{16}$ luego de la primera implantación exitosa de una asistencia ventricular izquierda (left ventricular assist device [LVAD]) con una duración de 10 días en un posquirúrgico de cirugía cardíaca. Poco después y luego del primer trasplante exitoso de corazón en 1967, se comenzaron a utilizar diferentes tipos de asistencia como puente a trasplante. El primer éxito con un corazón artificial total fue comunicado por Cooley et al. en 1969'16,17.

Debido a las complicaciones posquirúrgicas del trasplante, y con el apoyo del entonces recién formado National Heart and Lung and Blood Institute, se empezó a desarrollar en forma exponencial el campo de los DAV mecánica; es así como nacen los de primera generación. La década de los años 80 fue el acmé de los mismos, con el primer dispositivo de duración prolongada (112 días), el Jarvik-7 Total Artificial Heart (TAH). En el año 1984 se diseñaron nuevas bombas de asistencia de cámara única como puente a trasplante y con ellas se desarrolló la codificación de las distintas estrategias para su uso, lo que posibilitó lograr no solo el objetivo como puente a trasplante (bridge to transplant [BTT]) sino también como terapia de destino (destination therapy [DT]) y como puente a la recuperación (bridge to recovery [BTR]), como será explicado más adelante ${ }^{16,18}$.

Por lo anteriormente señalado, a partir del año 2003 los DAV dejaron de ser solo opciones terapéuticas para los pacientes elegibles para el trasplante, y comenzaron a utilizarse como DT. En el año 2006 nace la Interagency Registry of Mechanically Assisted Circulatory Support (INTERMACS) con el objetivo de ayudar a avanzar en la comprensión y aplicación de los DAV para mejorar la morbimortalidad y el estado de salud en pacientes con ICA ${ }^{16,18}$

Es por ello que INTERMACS ${ }^{19}$ ha definido siete perfiles clínicos en los pacientes con ICA antes de la implantación de un DAV, los cuales permiten la estratificación de los mismos en función de su perfil hemodinámico y el grado de daño de órgano blanco. Estos escenarios permiten mejor definición de la limitación y el grado de severidad de los pacientes ${ }^{1,2,19} \mathrm{y}$ se enumeran a continuación:

- Nivel 1. Shock cardiogénico, "crash and burn": pacientes hemodinámicamente inestables con hipoperfusión de órganos blanco a pesar de dosis máximas de inotrópicos y/o soporte mecánico.

- Nivel 2. Deterioro progresivo a pesar del soporte inotrópico, "sliding on inotropes": soporte inotrópico endovenoso permanente, manteniendo buena tensión arterial media (TAM) pero con un rápido deterioro de la función renal, estado nutricional o signos de congestión.

- Nivel 3. Estable pero dependiente de inotrópicos, "dependent stability": estabilidad hemodinámica con dosis bajas o intermedias de inotrópicos, necesarias para mantener TAM, evitar síntomas o falla renal.

- Nivel 4. Síntomas en reposo, "frequent flyer": paciente en clase funcional (CF) IV de la New York Heart Association (NYHA) ambulatorio, con síntomas recurrentes y sobrecarga de volumen.

- Nivel 5. Intolerancia al esfuerzo, "housebound": es también un paciente ambulatorio en CF IV de la NYHA que cesó toda actividad física, estable en el reposo pero con frecuente retención de líquido moderada y en algunos casos falla renal.
- Nivel 6. Esfuerzo limitado, "walking wounded": NYHA CF III. Limitación menor en la actividad física y ausencia de congestión en reposo. Se fatiga fácilmente con mínimos esfuerzos.

- Nivel 7. "Placeholder": paciente en CF III de NYHA sin desequilibrio en el balance hidrosalino actual o reciente.

Esta clasificación tiene el objetivo de unificar criterios en la descripción del estado clínico de los pacientes con ICA, optimizar la predicción de su riesgo perioperatorio y clarificar las indicaciones de cada una de las alternativas terapéuticas disponibles ${ }^{20}$. Actualmente la escala INTERMACS ha demostrado su valor para predecir mortalidad ${ }^{19,20} \mathrm{y}$ complicaciones posoperatorias ${ }^{21}$.

Esto fue analizado en un estudio retrospectivo por Barge Caballeroa et al. ${ }^{20} \mathrm{Al}$ utilizar los diferentes perfiles citados, observó el valor pronóstico posoperatorio en los pacientes con ICA trasplantados en carácter de urgencia. Como resultado del estudio se concluye que la mortalidad de los pacientes en el nivel 1 es tres veces superior que la de los pacientes en nivel 2 y cuatro veces superior que en aquellos en nivel 3 y 4 .

Con el avance tecnológico los DAV se fueron mejorando y desarrollando hasta la actualidad, en donde encontramos los de segunda y tercera generación con la aprobación del HeartMate // en el 2010, con más del $80 \%$ de supervivencia al año de la implantación y, con el nuevo corazón artificial bioprostético publicado recientemente por Carpentier et al. ${ }^{22}$ Actualmente las asistencias ventriculares se convirtieron en un paradigma en el tratamiento de la ICA pudiendo ser esta década la más prometedora para las mismas ${ }^{23}$.

Luego de este breve desarrollo histórico de la introducción de la asistencia ventricular en el tratamiento de la ICA, se describirán con más detalle las características, las ventajas y desventajas y los diferentes tipos de dispositivos disponibles.

\section{CLASIFICACIÓN Y HEMODINAMIA DE LOS DISPOSITIVOS DE ASISTENCIA VENTRICULAR}

Actualmente la industria se esfuerza por encontrar un dispositivo ideal, que sea durable, biocompatible, no trombogénico, resistente a la infección y de un costo razonable.

El primer dispositivo desarrollado fue el balón de contrapulsación intraaórtico (intraaortic balloon pump [IABP]) y continúa aún siendo el más comúnmente utilizado. Excede los propósitos de este trabajo su descripción pero básicamente fue diseñado para mejorar la relación entre el aporte y la demanda de oxígeno miocárdico. El IABP produce una modesta reducción de la sobrecarga ventricular, produce aumento de la TAM y del flujo coronario. Pero para su utilización los pacientes deben mantener algún grado de preservación de la función ventricular ${ }^{23}$.

Los nuevos DAV fueron diseñados para descargar de forma efectiva tanto el ventrículo izquierdo (VI) como el ventrículo derecho (VD). La sangre se obtiene de la aurícula izquierda o del propio VI, retornando a la aorta. Por consiguiente, se reducen las presiones y volúmenes intraventriculares, con reducción del estrés parietal y del consumo miocárdico de oxígeno ${ }^{24-26}$.

Si bien existen pocos estudios aleatorizados que comparan ambos tipos de asistencias, en un análisis de 117 pacientes con shock cardiogénico 


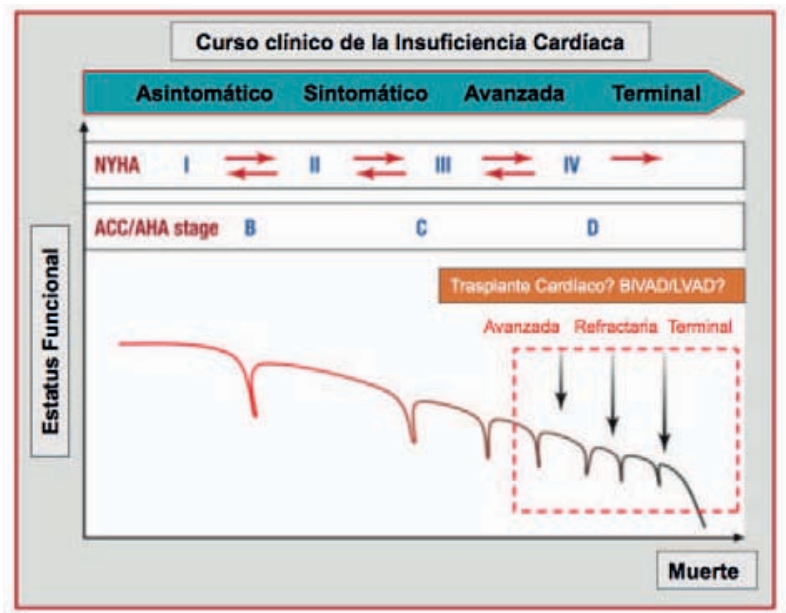

Figura 1. Curso Clínico de la insuficiencia cardíaca. Diferentes etapas y momentos oportunos para indicación de trasplante y/o dispositivos de asistencia ventricular. ACC: American College of Cardiology; AHA: American Heart Association; BiVAD: biventricular assist device (dispositivo de asistencia biventricular); LVAD: left ventricular assist device (dispositivo de asistencia ventricular izquierda); NYHA: New York Heart Association. Modificado de Tronchu JN. Left Ventricle assist devide: when and which patients should we refer?. Arch Cardiovasc Dis 2012;105(2):114-21.

refractario al IABP y/o vasopresores, Kar et al. ${ }^{27}$ observaron mejorías significativas en el índice cardíaco, la presión arterial sistólica, presión capilar pulmonar y función renal, con el uso de DAV durante un tiempo de implante promedio de 6 díass $^{28}$.

\section{DISPOSITIVOS DE PRIMERA GENERACIÓN}

Los primeros DAV fueron grandes sistemas de flujo pulsátil con una cámara de reservorio interna con válvulas de entrada y salida de flujo (Figura 2). Antes del año 2008 todas los DAV implantados en los EE.UU. usaban sistemas de flujo pulsátil con energía neumática (Abiomed BVS 5000, Thoratec VAD System, HeartMate 1000 IP LVAS, Berlin Heart, etc.) o eléctrica (Novacor N-100 LVAS, HeartMate 1000 XVE LVAS, LionHeart). Estos tenían excelente perfil hemodinámico y aumentaban la sobrevida pero con grandes limitaciones particularmente en cuanto a la duración a largo plazo, la necesidad de cirugías extensas para su colocación y largos cables extracorpóreos. Por estas características mencionadas eran más propensos a infección y la necesidad de una constitución corporal mediana a grande, un funcionamiento de la bomba audible y ruidoso, y con mayores complicaciones por ruptura del dispositivo hicieron que su uso decayera a lo largo de los años ${ }^{12,29}$ (Figura 3).

En el estudio Long-Term use of a left ventricular assist device for end stage heart failure del grupo Randomized Evaluation of Mechanical Assistance for the Treatment of Congestive Heart failure (REMATCH) $)^{30}$ la tasa de falla del dispositivo HeartMate 1000 XVE LVAS luego de 2 años fue del 35\% con una mortalidad del 10\%. Es así que desde que la Food and Drug Administration (FDA) aprobó el dispositivo HeartMate /l para BTT y luego para DT. el uso de los dispositivos de primera generación disminuyó desde el año 2007 de 316 implantes a 20 en 2013, representando los dispositivos de flujo continuo actualmente a más del $90 \%$ de las asistencias

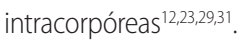

\section{DISPOSITIVOS DE SEGUNDA GENERACIÓN}

Estos dispositivos fueron desarrollados con el objetivo de mantener la asistencia ventricular a mediano y largo plazo. Son bombas de flujo con-

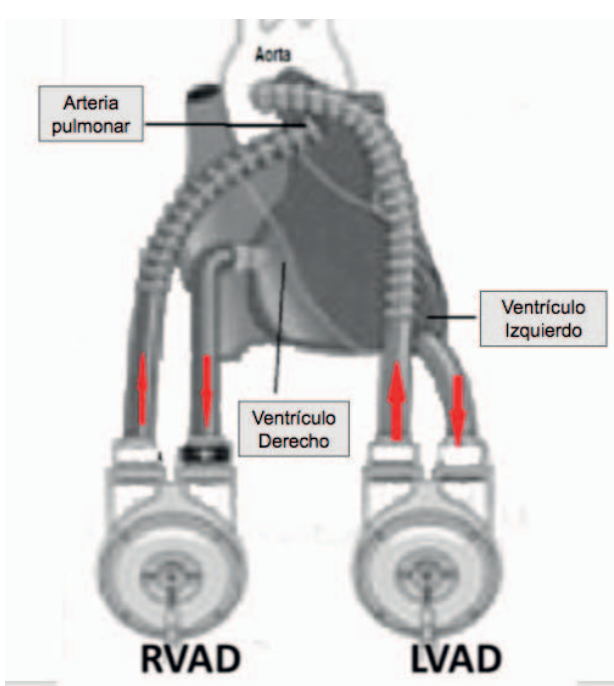

Figura 2. Esquema de Dispositivos de asistencia ventricular de primera generación. Uni o Biventriculares como en este caso. Extracorpóreos y de flujo pulsátil. RVAD: "right ventricular assist device" (disposirivo de asistencia ventricular derecha). LVAD: "left ventricular assist device" (dispositivo de asistencia ventricular izquierda). Extraída y traducida del inglés de: Ferrari M, Kruzliak P, Spiliopoulos K. An insight into short and long term mechanical circulatory support systems. Clin Res Cardiol 2015;104(2):95-111.

tinuo que tienen sólo una parte en movimiento, el rodillo o rotor interno, eliminando la cámara de reservorio y las válvulas de entrada y salida siendo por lo tanto, mucho más duraderas. Tienen un soporte de contacto que guía el movimiento y reduce la fricción entre las partes móviles e inmóviles. Son más pequeños, silenciosos y la implantación quirúrgica es menos traumática. Las bombas de flujo continuo también tienen líneas de conducción pequeñas, tienden a tener menores tasas de infección y pueden generar gastos de hasta $10 \mathrm{l} / \mathrm{min}$. Al eliminar las válvulas generan menos complicaciones tromboembólicas a 2 años y menores tasas de fallo del dispositivo $0^{12,29,31}$.

En aquellos pacientes en quienes se produjo falla del dispositivo, el tiempo medio para el intercambio del mismo o mal funcionamiento fue 539,7 días, aunque esto varía en las diferentes series ${ }^{32}$. La etiología principal de fallo reportada es la trombosis. Esto se explicaría por el lavado incompleto de la estasis sanguínea a nivel del soporte que se encuentra en íntimo contacto con el rodillo ${ }^{31}$.

Están controlados y alimentados desde consolas portátiles y se han utilizado en numerosos casos como terapia definitiva. Se ha conseguido supervivencias de 4 años ${ }^{12,29,31}$ (duración media de soporte ventricular izquierdo de 504,7 días), y la incidencia estimada de fallo del dispositivo fue relativamente baja, de 3,9\% en las diferentes series ${ }^{32}$

Los sistemas de flujo axial que emplean poseen una cánula con una turbina eléctrica en su interior que girando a gran velocidad, expulsa por inercia la sangre desde el VI a la aorta. Los dispositivos más utilizados y aprobados por la FDA son el el HeartMate I/ (Figura 4) y el Jarvik 2000

\section{Ila. HeartMate II}

El HeartMate ll fue el primero en ser aprobado por la FDA para DT y como BTT. Consta básicamente de tres partes: una bomba, cánulas de entrada y de salida, y un equipo controlador. El prototipo actual emplea un rotor axial de titanio con palas helicoidales que envuelven un eje central y un estator o circuito fijo, cuya función es asegurar un flujo laminar a la sali- 


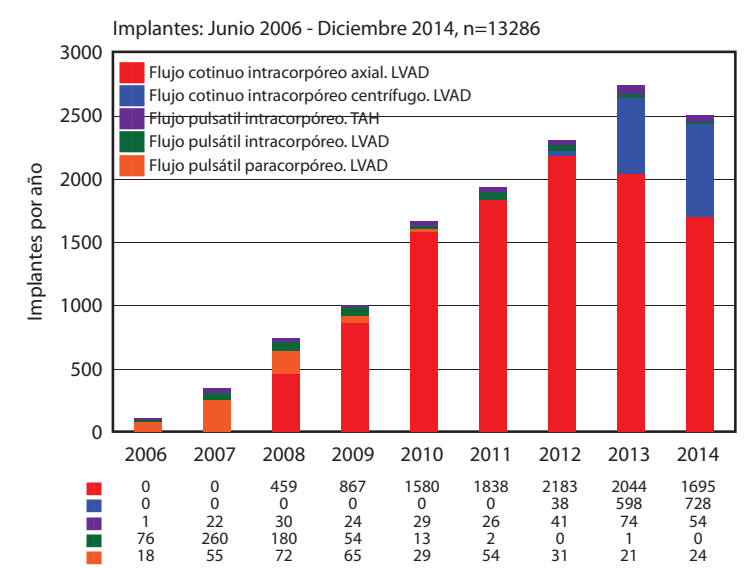

Figura 3. Distribución de dispositivos por tipo y año de implante. Se resalta el aumento del uso de los DAV de segunda generación (flujo axial) desde el año 2010 y el aumento del uso de los de tercera generación (centrifugos) a partir del 2013. LVAD: "left ventricular assist device" (dispositivo de asistencia ventricular izquierda). TAH: flujo pulsátil intracorpóreo. Modificado de: Kirklin JK, Naftel DC, Pagani FD, Kormos RL, Stevenson LW, Blume ED, et al. Seventh INTERMACS annual report: 15,000 patients and counting. J Heart Lung Transplant. 2015;34(12):1495-504.

da de la bomba. La bomba se coloca debajo del reborde costal izquierdo, la cánula de entrada es metálica de titanio, en ángulo recto y cuenta con un manguito de filtro para ser suturado al ápex del ventrículo izquierdo (Figura 4). Una única línea de conducción eléctrica sale a través de la piel hacia el controlador que ajusta la velocidad de la bomba en respuesta a la demanda fisiológica. En la actualidad se encuentra en desarrollo un sistema transcutáneo de trasmisión de energía con el objeti-

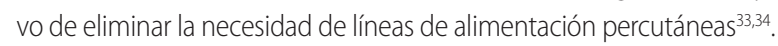

\section{Ilb. Jarvik 2000}

Es una bomba de flujo axial continuo que se caracteriza por ser un dispositivo silencioso, fácilmente implantable y portátil, consiste en una pequeña turbina que se sitúa en el ápex del VI, prácticamente encapsulada por el miocardio nativo. La sangre se impulsa a la aorta torácica descendente sin cánula de entrada ni válvulas, produciendo una corriente de alto flujo que lava continuamente la pequeña cámara a través de un tornillo de Arquímedes modificado que gira en el intervalo de las 1000 revoluciones por minuto, proporcionando un flujo continuo de hasta 10 litros por minuto. Todo ello reduce el riesgo de formación de trombos y hemólisis. La energía necesaria para su funcionamiento procede de una línea de conexión percutánea al sistema de baterías. La necesidad de anticoagulación en estos pacientes no está aún claramente establecida. Se ha utilizado en muchos casos como terapia definitiva, consiguiendo supervivencias de más de tres años ${ }^{35}$.

\section{DISPOSITIVOS DE TERCERA GENERACIÓN}

Son también dispositivos de flujo continuo que fueron diseñados con el objeto de mejorar el rendimiento, los aspectos mecánicos y la durabilidad de los DAV de segunda generación cuyo principal inconveniente es el desgaste por fricción en el soporte de contacto y el rodillo que tienen una vida útil (sumergida en sangre), de aproximadamente 5 años, siendo necesario en la DT una duración de aproximadamente 10 años ${ }^{31}$. Como consecuencia, se desarrollaron dispositivos de flujo continuo que utilizan bombas centrífugas más compactas, que no poseen soporte de contacto dado que utilizan la levitación magnética y/o hidrodinámica

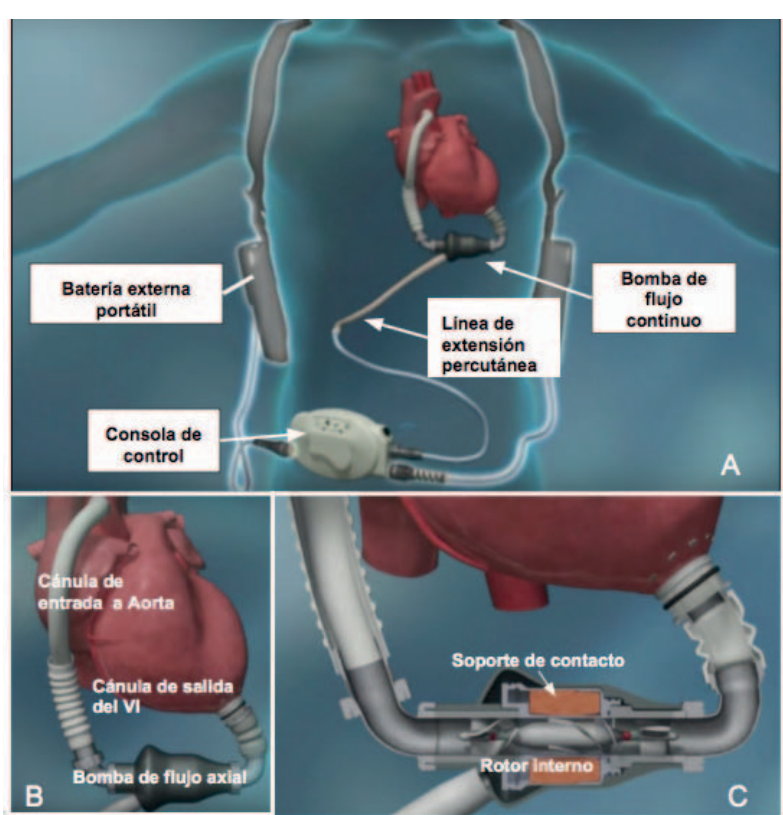

Figura 4. Heart Mate II. A: Ubicación intracorpórea del dispositivo, Baterías y consola externa. Unica línea de extensión percutánea. B y C: Conexiones. Nótese el rotor interno con las palas helicoidales en íntimo contacto con el soporte. VI: ventrículo izquierdo. Imagen extraída de video informativo en: http://heartmateii.com/heartmate-ii-system.aspx de @2014 Thoratec Corporation.

con esto se logra la suspensión del impulsor, las partes "flotan", no hay fricción, produciendo un menor desgaste en el rodillo. Además, este entorno libre de contacto está diseñado para optimizar la hemocompatibilidad. Al reducir el trauma sanguíneo mediante una suave manipulación del fluido con mayor flujo alrededor del impulsor que mejora el lavado, reduce la formación de trombos y de esta forma la necesidad de mantener valores elevados de Razón Normalizada Internacional (RIN) ${ }^{30,31}$

En cuanto a tamaño, son un poco más grandes que los de segunda generación. En algunos casos requieren de una inserción en un bolsillo a nivel abdominal, aunque se han diseñado dispositivos pequeños que pueden ser insertados en el espacio pericárdico. Si bien se espera que estos dispositivos duren por largos periodos todavía no se cuenta con estudios de seguimiento a más de 2 años 29,31 .

\section{Illa. HeartWare Ventricular Assist Device}

Es una bomba centrífuga miniatura (volumen de 50cc, pesa 160 gramos) que puede ser utilizada como LVAD (Figura 5) o biventricular (Biventricular assist device BiVAD) que utiliza un impulsor de hoja ancha (única parte móvil del dispositivo) suspendida por un sistema de soporte híbrido, magnético e hidrodinámico para evitar el contacto mecánico y el desgaste y así lograr un rendimiento óptimo, hemocompatibilidad y la fiabilidad a largo plazo. Es implantada en el espacio pericárdico y está diseñada tanto para pacientes internados como ambulatorios ${ }^{36,37}$. El Bridge to transplant HeartWare Left Ventricular Assist Device for the Treatment of Advanced Heart Failure (ADVANCE) study ${ }^{38}$ comparó 140 pacientes BTT sometidos al implante de un HeartWare, con 499 pacientes en un grupo de control de INTERMACS que eran en gran medida apoyados el HeartMate II. Las tasas de supervivencia a 1 año fueron 90,7\% en el grupo HeartWare y 90,1\% en el grupo control, lo que llevó a que la FDA apruebe el uso del HeartWare para los candidatos BTT y una expansión de las indicaciones más allá de las fronteras europeas. 


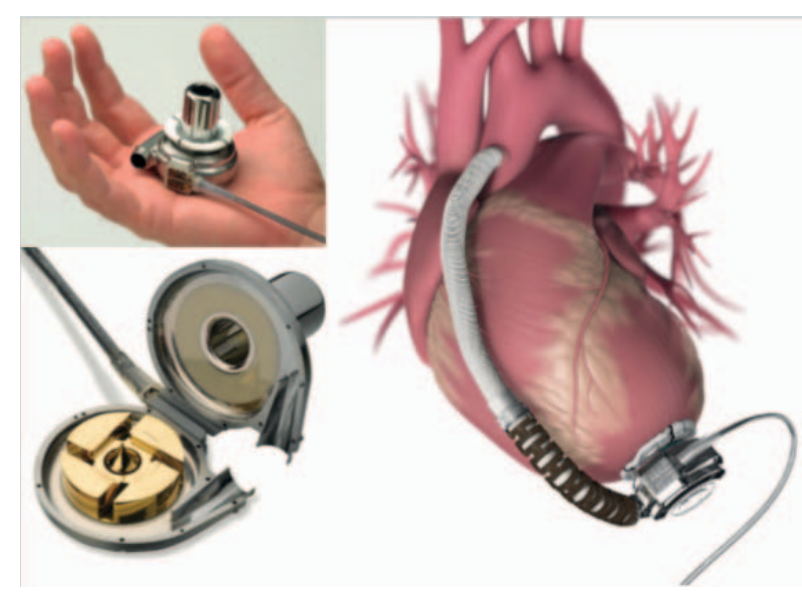

Figura 5. HeartWare LVAD: Mínimo tamaño de la bomba, con el impulsor de hoja ancha Implantado en el espacio pericárdico. Única línea de salida percutánea. Imagen de elaboración propia en base a: video informativo en https://www.heartware.com/products-technology/pump-design y Expert Rev Med Devices. $2013 \mathrm{Jul} ; 10$ (4):441-52.

En el estudio de no inferioridad ENDURANCE ${ }^{39}$ se compararon los resultados en los pacientes en DT en soporte con el HeartWare contra el actual líder de la industria, el HeartMate ll. Es un estudio multicéntrico prospectivo que incluyó 450 pacientes, los cuales fueron seguidos por 24 meses. Se randomizaron 2:1 a HeartWare o HeartMate II. Hacia el tercio final del estudio se realizaron cambios en el dispositivo HeartWare: en la cánula de flujo de entrada de la bomba, en el sistema de corte apical, y en las dosis antiplaquetarias y el régimen de anticoagulación (aspirina aumentó de 81 a 325 mg y el RIN de 2,0 - 3,0), lo que hizo suponer a los autores que durante dicha etapa se produciría una reducción de los eventos adversos. Se realizó una evaluación temporal para determinar el impacto de estos cambios. Se evidenció menor accidente cerebrovascu$\operatorname{lar}(\mathrm{ACV})$ hemorrágico y fallo ventricular derecho en el HeartMate ll. Pero estas diferencias se neutralizaron al analizar el grupo de pacientes ingresados luego de las modificaciones mencionadas. No hubo diferencias en cuanto al requerimiento de cambio de dispositivo en la cohorte completa $(89,7 \%$ vs $83,3 \%, p=0,066)$, pero fue significativamente menos frecuente en la cohorte HeartWare en el análisis del tercio final (96,3\% vs $85,0 \%, p=0,026)$. Los autores concluyeron que las mejoras en el dispositivo conllevan a una selección optimizada de los pacientes, a menos tasas de complicaciones y menor requerimiento de recambio. Así mismo resulta necesario para evitar mayores eventos de sangrado fatales y no fatales el ajuste óptimo de los fármacos antitrombóticos ${ }^{39}$.

\section{IIIb. Heart Mate III}

El HeartMate III (Figura 6) es un dispositivo de flujo continuo con una bomba centrífuga con un rotor con doble función: motora y de levitación magnética rotacional y radial. Ambas se logran en una sola unidad integrada que incorpora todos los componentes electrónicos de control en la carcasa inferior del dispositivo, lo que resulta en un rendimiento eficiente y compacto. Los grados de libertad. movimiento axial y de inclinación, se consiguen mediante soporte magnético pasivo sin consumo de energía ${ }^{31,33}$. El dispositivo continúa en evaluación y está pendiente su aprobación.

\section{SOPORTE BIVENTRICULAR}

Actualmente se cuenta con la aplicación del HeartWare como BiVAD y del SynCardia TAH (Figura 7). Este es solo para pacientes con superficie

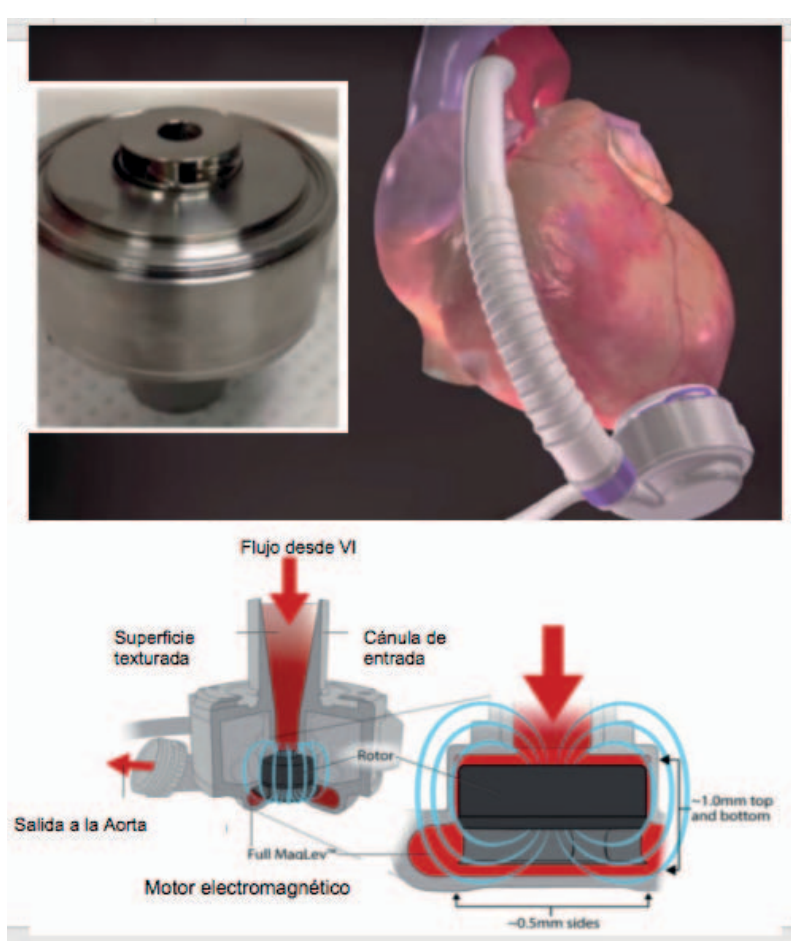

Figura 6. HeartMate III LVAD con sus diferentes partes: motor electromagnético y cánulas de entrada y salida. VI: ventrículo izquierdo. VI: ventrículo izquierdo. Imagen de elaboración en base a: Netuka I, BeyersdorfF, Garbade J, Krabatsch T, Marasco S, Morshuis M, et al. HeartMate 3 fully magnetically levitated LVAD for the treatment of advanced heart failure: results from the CE Mark Trial. Presented at the 19th Annual Meeting of the Heart Failure Society of America (HFSA); 2015

corporal de más de $0,7 \mathrm{~m}^{2}$ y consiste en dos ventrículos semirrígidos cubiertos con cuatro diafragmas flexibles de poliuretano que separa las cámaras sangrientas de las cámaras de aire de poliuretano. Está aprobado para su uso en los EE.UU., Canadá y Europa. Se implantó por primera vez en un paciente en la Universidad de Arizona en 1988, y más de 1.100 pacientes ya han recibido el apoyo de la SynCardia TAH siendo el soporte más largo de 1374 días antes del trasplante de corazón con éxito ${ }^{24}$. Se encuentra en estudio el corazón bioprotésico de Carpentier et al. ${ }^{22}$ el CARMAT TAH, con solo 2 pacientes reportados. Es una bomba pulsáti que usa energía electrohidráulica. Todos los componentes están incorporados en un solo dispositivo colocado en el saco pericárdico después de la escisión de los ventrículos nativos. Promete ser biocompatible y tener un comportamiento más fisiológico.

\section{EVALUACIÓN PARA EL IMPLANTE DE LOS DAV}

\section{INDICACIONES}

En los pacientes con ICA, los avances tecnológicos han facilitado el uso de los dispositivos de asistencia ventricular tanto izquierda o derecha como biventricular, implantables quirúrgicamente como puente a la recuperación, puente para el trasplante, para su uso como terapia permanente (destino) y, actualmente puente a la candidatura (bridge to candidacy $[\mathrm{BTC}])^{40,41}$.

Según se desprende de lo analizado previamente, independientemente de la causa que lleve al implante de un dispositivo, como principales beneficios se busca:

- Mantener una adecuada perfusión de órganos vitales. 


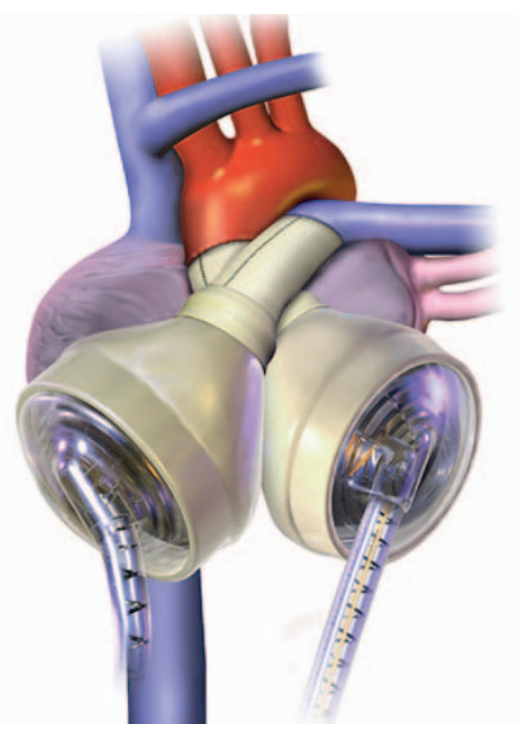

Figura 7. Syncardia Total Artificial Heart. 2 ventrículos semirrígidos cubiertos con cuatro diafragmas flexibles de poliuretano. Imagen extraída de: www.syncardia.com

- Disminuir las presiones intraventriculares y así bajar la congestión pulmonar.

- Disminuir los volúmenes ventriculares, el estrés parietal y el consumo de oxígeno.

- Aumentar la perfusión coronaria.

- Apoyo circulatorio durante intervenciones cardíacas y electrofisiológicas complejas.

La guía de IC de la Sociedad Europea de Cardiología 2016 divide las indicaciones según las características clínicas de los pacientes:

1) Pacientes potencialmente elegibles para la implantación del $L V A D$, refiriéndose a pacientes con más de 2 meses de síntomas severos a pesar de tratamiento médico óptimo, TRC o colocación de CDI, y alguna de las siguientes:

- $\mathrm{FEVI}<25 \%$ y consumo máximo de oxígeno $<12 \mathrm{ml} / \mathrm{kg} / \mathrm{min}$.

- Más de 3 hospitalizaciones en los últimos 12 meses sin una causa precipitante.

- Dependencia de inotrópicos endovenosos.

- Disfunción progresiva de órgano blanco (empeoramiento del daño renal o hepático) debido a mala perfusión y no a presiones inadecuadas del llenado ventricular (presión capilar pulmonar $>20$ mmHg, presión sistólica <80-90 o índice cardiaco <2 l/min/m²).

- Ausencia de disfunción ventricular derecha junto con insuficiencia tricuspídea severa.

2) Recomendaciones (Clase de evidencia lla. Nivel C) para la implantación de LVAD en paciente con ICA:

- Un LVAD debe ser considerada en pacientes que tienen IC con deterioro severo de la FEVI en fase terminal a pesar del tratamiento médico y el dispositivo óptimo y que son elegibles para el trasplante de corazón con el fin de mejorar los síntomas, reducir el riesgo de hospitalización por insuficiencia cardíaca y el riesgo de muerte prematura (BTT).

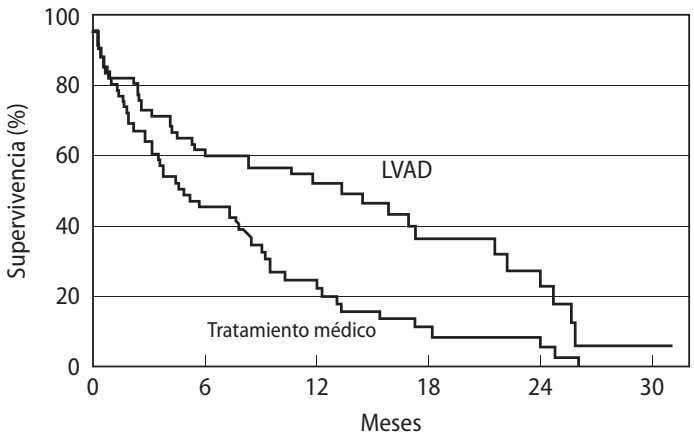

№ en riesgo

$\begin{array}{lllllll}\text { LVAD } & 68 & 38 & 22 & 11 & 5 & 1\end{array}$

$\begin{array}{lllllll}\text { Trat. médico } & 61 & 27 & 11 & 4 & 3 & 0\end{array}$

Figura 8. Curva de Kaplan-Meier. LVAD: left ventricular assist device (dispositivo de asistencia ventricular izquierda). Modificado de: Rose EA, et al. Long-term use of a left ventricular assist device for end-stage heart failure. Randomized Evaluation of Mechanical Assistance for the Treatment of Congestive Heart Failure (REMATCH). N Engl J Med 2001;345(20):1435

- Un LVAD debe ser considerada en pacientes IC con deterioro severo de la FEVI en etapa terminal a pesar del tratamiento médico y el dispositivo óptimo y que no son elegibles para el trasplante de corazón para reducir el riesgo de muerte prematura (DT).

Según las variables mencionadas del INTERMACS ${ }^{19}$ y las recomendaciones de las guías arriba mencionadas existen diferentes contextos clínicos para la indicación de los DAV.

\section{la. Puente a trasplante}

Es uno de los principales escenarios en el cual se propone el uso de los DAV en aquellos pacientes seleccionados con ICA que serían candidatos a trasplante y en los cuales no hay disponible un donante. En los EE.UU., la indicación BTT se realiza para estabilizar la circulación hemodinámica en pacientes con muy mala función cardiaca, para lograr una mejor perfusión y función de órganos nobles al momento del trasplante 25,29 .

En estos pacientes se utilizan fundamentalmente las asistencias pulsátiles, tanto en situación intra como paracorpórea. Los mejores resultados se obtienen en series en las que el implante es precoz y se realiza en forma electiva, contrario a lo observado en los casos indicados en carácter de urgencia ${ }^{42}$.

La evidencia y el uso aún siguen siendo controvertidos. En EE.UU. se realizó un estudio prospectivo en 5 centros en el que se comparó la seguridad y la eficacia del sistema Syncardia CardioWest como puente a trasplante en 81 pacientes con IC avanzada, contra un grupo histórico de características balanceadas. Era una población en CF IV de la NYHA, con un índice cardíaco $<2 \mathrm{l} / \mathrm{min} / \mathrm{m}^{2}$, tensión arterial sistólica $<90$ mmHg o una presión venosa $>18$ mmHg bajo tratamiento inotrópico o IABP. El 79\% de los pacientes del grupo con DAV se trasplantó con éxito y con diferencia significativa respecto al otro grupo en el que fue del $46 \%(p<0,001)$. Al año la diferencia en la supervivencia fue del 70\% versus el 31\%, significativo también para el grupo tratamiento ${ }^{12}$

En otra revisión retrospectiva realizada en el Hospital Universitario de Pensilvania entre los años 1995-2007; se analizaron 266 pacientes que recibieron: LVAD, BiVAD programada y un tercer grupo en donde se debió implantar un BiVAD de urgencia. Al observar los resultados en los que eran pacientes en plan de trasplante cardíaco $(n=141)$ aquellos que recibieron LVAD en el 79\% fueron trasplantados sin diferencias significa- 


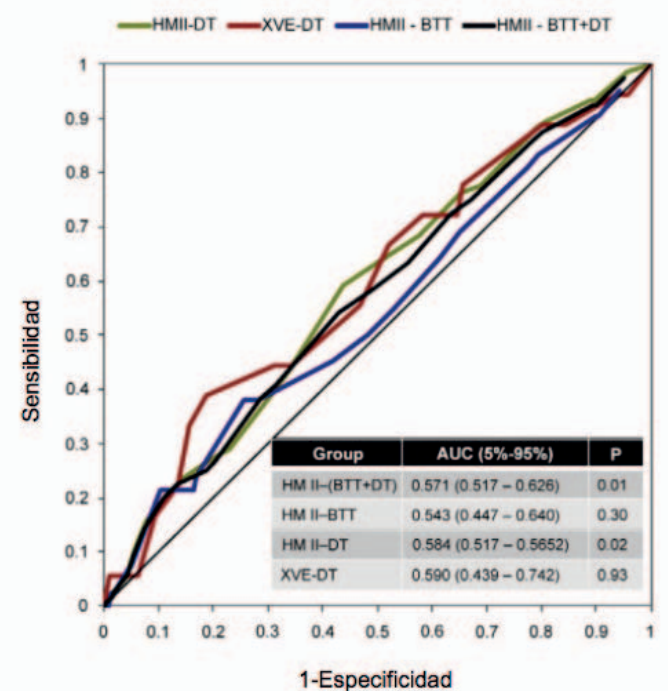

Figura 9. Curva ROC que evidencia moderada sensibilidad del Destination Therapy Risk Score. HM II: Heart Mate II. BTT: "bridge to trasplantationt" (puente a trasplante). DT: "destination therapy" (terapia de destino). XVE Modificado de Teuteberg JJ, Ewald GA, Adamson RM, Lietz K. Miller LW, Tatooles AJ et al. Risk assessment for continuous flow left ventricular assist devices: does the destination therapy risk score work? An analysis of over 1,000 patients.J Am Coll Cardiol. 2012;60(1):44-51

tivas con los que presentaron BiVAD programada (65\%). Si presentaron diferencias significativas en sobrevida al comparar el grupo de LVAD vs. BiVAD de urgencia (10/22 $p=0.0009)$. Estos resultados se mantuvieron al analizar la tasa de egreso hospitalario y sobrevida extrahospitalaria. Por lo que los autores destacan que en este grupo de pacientes es importante la decisión inicial más que el tipo de asistencia elegida ${ }^{41}$

\section{Ib. Terapia de destino}

En pacientes seleccionados con ICA que no son aptos para trasplante cardíaco, reciben DAV como DT para mejorar no solo la sobrevida sino también la calidad de vida y los síntomas. Ésta estrategia ha constituido el mayor porcentaje de pacientes con LVAD desde $2011^{29}$.

En el estudio REMATCH ${ }^{30}$ se validó el beneficio de los DAV en 129 pacientes con ICA con contraindicaciones para el trasplante cardíaco. Los criterios de inclusión fueron la presencia de CF IV durante al menos 90 días, FEy $<25 \%$ y un consumo de oxígeno $<12 \mathrm{ml} / \mathrm{kg} / \mathrm{min}$ o la necesidad de tratamiento inotrópico. Fueron asignados al azar para recibir tratamiento médico óptimo o un HeartMate I LVAD como DT. La supervivencia a un año en el grupo de asistencia ventricular izquierda fue de 52\% comparado con el $25 \%$ en el grupo de terapia médica óptima $(p=0,002)$, a dos años la supervivencia fue del $23 \%$ comparado con el $8 \%$ en el grupo de terapia médica $(p=0,09)$. En general, la mortalidad por todas las causas se redujo en un 48 por ciento mediante la inserción de la asistencia ventricular izquierda (Figura 8). Hubo una mejoría de la CF y las mediciones de la calidad de vida mejoraron de forma significativa a un año de seguimiento en el grupo de asistencia ventricular izquierda. La IC fue la principal causa de muerte en el grupo tratamiento médico, mientras que en grupo LVAD lo fueron la sepsis y la falla del dispositivo ${ }^{30}$.

Estos hallazgos son consistentes en todas las series y como ya se mencionó anteriormente las nuevas tecnologías aplicadas a DT como en el estudio ENDURANCE ${ }^{39}$ con el HeartWare y el HeartMate II, refieren mejoría de la sobrevida, la calidad de vida, y menor impacto psicológico en los pacientes. Esto sugiere que los beneficios de la restauración de un perfil hemodinámico relativamente normal podrían superar el riesgo quirúrgico y las complicaciones posteriores ${ }^{34,39,42}$

Como principales candidatos a esta indicación se han propuesto los pacientes con IC con más del 50\% de mortalidad esperada al año y que no sean candidatos a trasplante: pacientes en shock cardiogénico, con IC crónica con bajo gasto y daño de órgano blanco, dependientes de inotrópicos en CF IV, pacientes con IC crónica con caquexia cardíaca, consumo de $\mathrm{O}_{2}$ máximo $<10 \mathrm{ml} / \mathrm{kg} / \mathrm{min}$, hiponatremia o insuficiencia renal progresiva ${ }^{12}$

\section{Ic. Puente a la recuperación}

Los DAV en combinación con terapias optimizadas pueden, al desprecargar el VI, inducir la recuperación del miocardio en un subgrupo de pacientes con miocardiopatía no isquémico necrótica ${ }^{41}$. Si luego de la evaluación del paciente y de su función ventricular a bajo o mínimo soporte mecánico hay pruebas de significativo remodelado reverso miocárdico, estos paciente en terapia "puente a la recuperación" pueden ser considerados para el retiro de la asistencia ${ }^{43}$. Hay reportes de casos con exitosa explantación del dispositivo y recuperación completa de la fracción de eyección ventricular izquierda $(\mathrm{FEVI})^{44}$.

Birks et al. ${ }^{45}$ reportaron $70 \%$ de explantes del Heartmate VAD en una serie de 15 pacientes tratados con IECA, BB, ARA, espironolactona y clenbuterol. Esta cohorte tuvo una tasa de supervivencia de $82 \%$ a 4 años y e 88\% evolucionaron libres de IC recurrente. Todavía está en estudio la forma de weaning del LVAD. La combinación de la terapia agresiva médica, descarga del VI y el programa de vigilancia cuidadosa podría ser una estrategia con excelentes resultados a corto y medio plazo.

En la práctica, esta indicación es minoritaria, representa sólo el 6\% de los DAV implantados entre el 2003-2004 en un Registro Internacional ${ }^{46}$ En este escaso porcentaje se observó explantación en el 50\% de los casos, con una supervivencia a largo plazo de entre 25 y 30\%. Varios estudios observacionales y registros han intentado buscar predictores clínicos, hemodinámicos, ecocardiográficos, neurohormonales, anatomopatológicos capaces de identificar aquellos pacientes que podrían presentar recuperación ventricular, sin encontrar ninguno significativo hasta el momento 44,46

Las principales indicaciones para esta estrategia son: miocarditis aguda, fallo agudo primario del injerto postrasplante y miocardiopatía periparto, además del shock cardiogénico?.

\section{Id. Puente a la candidatura}

Desde que el trasplante cardíaco es una opción más realista después de la terapia con DAV en EE.UU., se utilizan en los casos en los que la indicación de trasplante no es claro, como "puente a la candidatura" bajo la indicación de DT. Esta opción da tiempo para investigar la idoneidad de trasplante, mientras se estabiliza y, a menudo se mejoran los parámetros críticos como el aumento de la resistencia vascular pulmonar, un marcador de riesgo de falla ventricular derecha luego del trasplante ${ }^{27}$. Por lo tanto, se indica habitualmente a pacientes con ICA o compromiso de órgano blanco con contraindicaciones relativas al trasplante en los cuales el perfil hemodinámico podría mejorar pudiendo considerarse de nuevo la opción del trasplante o de lo contrario la DT 1,47.

Además, los DAV pueden ser una opción aceptable para pacientes con una enfermedad oncológica reciente que pueda ser curada y 
en quien no se llegaría a cumplir los 5 años libres de enfermedad requeridos típicamente para el trasplante ${ }^{1,2,47}$.

\section{II. ¿CUÁL ES EL MOMENTO OPORTUNO PARA LA COLOCACIÓN DE UN DAV?}

En la IC aguda los pacientes sin disfunción cardíaca previa que requieren soporte hemodinámico se someten a DAV como "tratamiento de rescate". El enfoque actual en muchos centros es estabilizar la circulación y mejorar la perfusión de los órganos blanco ${ }^{41}$ con estrategias como la oxigenación por membrana extracorpórea (ECMO) o IABP o bombas de circulación extracorpórea, al intentar recuperar la función del corazón con revascularización o tratamiento médico. Los pacientes que no logran una recuperación temprana de la FEVI pueden ser considerados para un DAV de larga duración, predominantemente izquierdo (LVAD) o BiVAD'. El registro INTERMACS muestra mejores resultados en los pacientes implantados con un nivel INTERMACS 4 a 7, aunque la mayoría se realizan casi en un 60\% en los niveles 1 a $3^{29,48}$. La colocación temprana del DAV (INTERMACS 4 a 7), se evaluó en un ensayo reciente que reveló mejores resultados que en aquellos pacientes que continuaron con el tratamiento médico ${ }^{49}$.

Por lo general, los pacientes con IC avanzada considerados para DAV dado el estadio avanzado de la enfermedad, pueden estar con soporte inotrópico continuo o manifestar una disminución de la función de los órganos diana. Se quejan principalmente de limitaciones en su vida diaria, con disnea y fatiga presentes en reposo o durante el mínimo esfuerzo, lo que les confina a la cama o a permanecer en el hogar. Para los pacientes ambulatorios, las salidas son excursiones temidas y raras, y caminar es limitado. Padecen episodios repetitivos de retención de sal y agua o episodios de bajo gasto cardiaco lo que los lleva a las reinternaciones frecuentes (Figura 1). Todo lo mencionado con anterioridad, ocurre a pesar de de la optimización del tratamiento ${ }^{2,50}$.

Si bien, como fue comentado, en el estadío D de la ICA se han asociado otras terapias (CDI y TRC) por sobre el tratamiento médico, la evolución es hacia un progresivo deterioro con respuesta transitoria o escasa. Con el avance de la enfermedad, incluso la titulación de fármacos basados en la evidencia se dificulta debido a la hipotensión, insuficiencia renal, debilidad profunda, entre otras comorbilidades?

La evolución clínica se asocia al deterioro hemodinámico, por lo que es crucial no solo controlar la FEVI, sino también los diámetros sisto/diastólicos, la presencia de insuficiencia mitral y llevar a cabo una evaluación completa de la función del VD, ya que su disfunción es un factor pronóstico determinante ya que aumenta en gran medida la mortalidad perioperatoria ${ }^{50}$.

Por consiguiente, los pacientes con ICA deben ser referidos en forma temprana a los equipos de IC experimentados con el fin de discutir la terapia, evaluar el pronóstico y organizar un plan de atención que permita la implantación electiva de un LVAD si es necesario y adelantarse a la evolución natural con mal pronóstico de la enfermedad.

\section{CONTRAINDICACIONES:}

Las contraindicaciones absolutas incluyen enfermedad sistémica con una expectativa de vida de menos de 2 años, o malignidad en los próximos 5 años, disfunción renal o hepática terminal, enfermedad pulmonar obstructiva crónica (EPOC) severa u otra enfermedad con falla multiorgánica (FMO) irreversible, ACV reciente, desorden psiquiátrico que pueda comprometer la adherencia al tratamiento? Contraindicaciones relativas que pueden mejorarse y replantear la colocación: problemas en las pruebas de coagulación, hemorragia no controlada, sepsis no controlada, o infección por virus de inmunodeficiencia humana.

La edad del paciente no es una contraindicación formal y dependiendo del estado general del paciente y la presencia de comorbilidades. La implantación de la asistencia mecánica puede ser propuesto en pacientes $>65$ años ${ }^{29,51}$. Adamson et al.52 evaluaron el uso de HeartMate // en la población mayor de 70 años y observaron no solamente aumento de la sobrevida sin diferencias significativas con respecto a la población de menor edad sino también una mejoría significativa de la calidad de vida en ambos grupos, lo que ampliaría las opciones a un grupo excluido del trasplante.

La hipertensión venocapilar pulmonar no es una contraindicación si la función del VD está conservada o levemente deprimida. Así como tampoco lo son una toracotomía o válvulas mitrales mecánicas previas o insuficiencia aórtica. Sólo llevarán a modificar la ruta de acceso y llevarán al recambio de válvula por una biológica y a la reparación aórtica en la misma cirugía².

Los factores psicosociales desempeñan un papel importante en la evaluación de la candidatura para el DAV. Los dispositivos implantados requieren una vigilancia constante para reconocer y manejar las complicaciones. Los pacientes sin estructuras de apoyo adecuadas y aquellos cuya vigilancia puede verse afectada por la enfermedad psiquiátrica, disfunción cognitiva o la adicción a las drogas o el alcohol no son buenos candidatos, tema que será ampliado posteriormente.

\section{PREIMPLANTE DEL DISPOSITIVO}

Como vimos anteriormente, muchos factores relacionados al paciente pueden disminuir la sobrevida post implante del DAV e incluso al coexistir varias comorbilidades pueden hacer que no sea un candidato adecuado para la colocación de los mismos. La fragilidad preoperatoria es un factor asociado a mayor mortalidad posoperatoria y readmisión hospitalaria ${ }^{53}$.

Por ello, en los pacientes que tendrían indicación de colocación del DAV la evaluación preimplante es fundamental y se basa en detectar aquellos parámetros que estén relacionados con peores desenlaces luego de la implantación del dispositivo ${ }^{54}$. Esto requiere la evaluación del paciente según la gravedad de la IC, el riesgo quirúrgico, la estabilidad psicosocial, y la capacidad de adherirse al régimen de auto-cuidado post colocación del DAV. El médico tratante debería ser capaz de identificar y comunicar cualquier condición o comorbilidad al equipo de IC y, dar todas las opciones disponibles al paciente ${ }^{55}$.

\section{ROL DE LOS SCORE DE RIESGO EN ICA}

En la evaluación cardiovascular del riesgo múltiples variables han sido asociadas a mayor mortalidad en pacientes con IC, es por eso que cobran importancia los diferentes scores que permiten estratificar a los pacientes y predecir la mortalidad antes y después de la colocación del dispositivo ${ }^{47}$

El Seattle Heart Failure Risk score $e^{56}$ establece diferentes variables que permiten predecir la evolución hospitalaria, la estadía y la supervivencia al 
año. Entre ellas: edad, sexo, CF de la NYHA, el peso, la FEVI, la presión arterial sistólica, la presencia de cardiopatía isquémica, furosemida diaria equivalente de dosis, el uso de inotrópicos, estatinas, allopurinol, tratamiento médico de IC, CDI, parámetros de laboratorio (hemoglobina, porcentaje de linfocitos, ácido úrico, colesterol sérico, y el sodio sérico). El mismo fue validado para los pacientes en plan de colocación de LVAD en el año 2010 al agregarle dos variable: uso de IABP, uso de inotrópicos ${ }^{57}$.

Por otro lado, Ketchum et al..$^{57}$ identificaron correctamente qué pacientes tenían un riesgo lo suficientemente alto como para requerir la implantación del LVAD, y qué grupo, por no depender de inotrópicos y ser ambulatorios, podrían beneficiarse con la colocación programada con el uso del mismo score

La puntuación de Lietz-Miller fue desarrollada a partir de un registro de pacientes que recibieron soporte como DT. El Destination Therapy Risk Score basado en parámetros de laboratorio y variables clínicas fue capaz de identificar a aquellos que presentan un riesgo relativamente bajo en comparación a los que tienen mayor riesgo de mortalidad. Inicialmente fue evaluado en paciente con dispositivos de primera generación (HeartMate XVE), y luego se validó tomando una cohorte de más de 1.000 pacientes con HeartMate II. El score logró discriminar entre aquellos con alta y baja mortalidad hospitalaria a los 90 días, pero no pudo distinguir entre los grupos de bajo y medio riesgo. El receiver-operator characteristic curves (ROC) demostró solo modesta capacidad para discriminar riesgo de mortalidad hospitalaria a los 90 días en pacientes con LVAD flujo continuo (Figura 9) ${ }^{58}$.

Las puntuaciones desarrollados para evaluar la función de órganos extra cardíacos también se aplicaron a la evaluación pre implante de los DAV. El Modelo de la Enfermedad Hepática en Fase Terminal (MELD) ${ }^{59}$ ha demostrado ser un predictor de eventos adversos en INTERMACS ${ }^{60}$ y estos hallazgos fueron validados por Yang et al. ${ }^{61}$ en un estudio de 200 pacientes.

Los scores de riesgo ganan interés especialmente en las personas mayores. Utilizando la base de datos de ensayos clínicos HeartMate // de 1.122 pacientes, Cowger et al (64) desarrolló un score de riesgo para los pacientes considerados para asistencias ventriculares de flujo continuo, dado de que los scores previos se realizaron con los antiguos dispositivos de flujo pulsátil. Los componentes del HeartMate II multivariable risk score (HMRs) incluyeron la edad (década), la albúmina sérica (mg/dl), la creatinina sérica (mg/dl), el RIN, y la experiencia del centro aumentando el riesgo anual con un volumen del centro menor a $15^{62}$.

En segundo lugar, la falla del VD resulta en un mal llenado del VI y por lo tanto, menor flujo de salida en la LVAD. La falla del VD pre implante está asociada a mayor mortalidad, mayor riesgo de sangrado y de re operación, mayor tiempo de estadía hospitalaria y mayores tasas de insuficiencia renal ${ }^{62}$

Matthews et al. ${ }^{63}$ desarrollaron un score para evaluar principalmente falla de VD, y determinar qué variables clínicas y de laboratorio se asocian a su presencia prediciendo mortalidad posoperatoria en pacientes con terapia de destino. Los investigadores encontraron como predictores independientes el uso de vasopresores, aspartato aminotransferasa por encima de $80 \mathrm{UI} / \mathrm{l}$, valores de bilirrubina mayores a $2 \mathrm{mg} / \mathrm{dl}$, y niveles de creatinina mayores a 2,3 mg/dl. El área bajo la curva del score fue de $0,73 \pm 0,04$.

\section{EVALUACIÓN DE FRAGILIDADY ASPECTOS PSICOSOCIALES}

Al igual que los candidatos a trasplante los pacientes candidatos a un DAV deben ser evaluados por profesionales de la salud mental y asistentes del servicio social para asegurar los cuidados postoperatorios y la adherencia al tratamiento antes de la decisión de colocación del mismo ${ }^{40}$. Muchas veces las largas listas de espera generan, en los pacientes internados en plan de trasplante, tanto depresión como aislamiento socia lo que conlleva peor pronóstico independientemente de los factores biomédicos. En un estudio prospectivo ${ }^{64} \mathrm{y}$ en el análisis extendido del Waiting for a New Heart Study ${ }^{65}$ se vio la importancia de los factores de riesgo psicosociales los cuales se asociaron a un peor pronóstico al momento del trasplante y un mayor requerimiento de DAV en aquellos que se encontraban en lista de espera. Los pacientes refirieron gran estrés a mantenerse aislados, a ser separados de las personas de confianza, miedo al estar solos durante la espera del órgano o al recibir la llamada el día del trasplante. Dichas preocupaciones denotan problemas emocionales y ambivalentes frente a la situación del trasplante, produciendo progresión de la enfermedad y deterioro físico y hemodinámico.

En algunos casos el principal síntoma es la ansiedad extrema y no la depresión, siendo en algunos casos más prevalente la ansiedad en el preoperatorio y la depresión en el postoperatorio ${ }^{65,66}$.

Si bien el período posterior a la colocación de un DAV es de adaptación, uno de los efectos esperados es la mejoría de calidad de vida y la capacidad funcional y, con ello la disminución de los síntomas de depresión y ansiedad. En el estudio de Reynard et al. ${ }^{67}$, se investigó el curso de estos síntomas en una cohorte de pacientes ambulatorios con LVAD y se vio que ambos, ansiedad y depresión, mejoraron después de la colocación del dispositivo y se mantuvieron estables por lo menos un año.

Esto es importante para el seguimiento puesto que tanto la depresión como los trastornos de ansiedad, el consumo de drogas ilegales y de alcohol, se han visto relacionados con un aumento de la morbimortalidad, mayores readmisiones hospitalarias, mayores tasas de infecciones del dispositivo principalmente debido a una disminución del cuidado personal y la mayor tendencia a no ser adherentes al tratamiento ${ }^{40,68-70}$

Otra observación importante de mencionar es que luego del primer período, los pacientes suelen regresar a sus actividades habituales y roles sociales, a tener más confianza en su autocuidado, reconocen los problemas y limitaciones de sus dispositivos y, potencialmente, hacen frente a los síntomas de IC y a los relacionadas con el DAV71. Si bien aún, el trasplante hoy en día continúa siendo el mejor tratamiento y más efectivo en cuanto a los alcances en la mejoría de calidad de vida ${ }^{72}$.

Dado que la carga emocional luego del egreso hospitalario en este tipo de pacientes es alta, involucra al núcleo familiar, requiere preciso cuidado en las prescripciones médicas, reconocer los signos de alarma, concurrir a la rehabilitación cardiovascular, entre otros, se ha propuesto en los últimos años la integración al equipo de IC de médicos especialistas en cuidados paliativos ${ }^{40,73}$

Un manejo progresivo por parte del especialista en cuidados paliativos puede dirigir la educación y la comunicación tanto a la familia, cuidadores y paciente, ayudando a comprender mejor su enfermedad, las opciones de tratamiento y el pronóstico, y acompañar en la toma de decisiones médicas. Es por eso que estos pacientes tan complejos deben ser seguidos por 
un típico equipo multidisciplinario compuesto por médicos, enfermeras, trabajadores sociales, fisioterapeutas y psicólogos, todo lo cual genera mejor satisfacción, comunicación y reduce el estrés y la ansiedad ${ }^{73,74}$.

Por otro lado, el cuidador, normalmente un miembro de la familia u otro allegado, debe administrar un régimen de medicación compleja, cambiar los apósitos estériles, monitorear los signos vitales del paciente y pesos, y aprender el funcionamiento del LVAD. La sobrecarga de los cuidadores puede ser significativa. En los primeros meses, muchos acompañantes describen "un sentido abrumador de miedo y ansiedad"75. Es por ello que cobra especial importancia el equipo de salud tanto para la educación, la preparación y el soporte emocional del paciente y su entorno familiar.

\section{COMPLICACIONES Y COSTO/EFICACIA DE LOS DAV}

Son múltiples las complicaciones que pueden llegar a presentarse luego de la implantación de los dispositivos. En una revisión sistemática de 40 estudios $^{76}$ se vio que a los 12 meses de colocación, los pacientes sufrieron una variedad de complicaciones graves. Los estudios informaron amplios rangos para los eventos adversos: 4-27\% hemorragia que requirió transfusión siendo la hemorragia gastrointestinal la más frecuente con una prevalencia de $22 \%$ en los pacientes con LVAD33 1,5-40\% de ACV; infección 3,3-48\%; fallo del dispositivo 1-14\%; 3-30\% de IC; $11-32 \%$ de reintervenciones; e insuficiencia renal $3-53 \% 76$

En el último registro de INTERMACS ${ }^{29}$ la curva de supervivencia para dispositivos implantados de flujo continuo desde el año 2008 muestra una supervivencia a 1 año en su conjunto del $80 \%$ y 2 años de supervivencia del 70\%. En el mismo registro los eventos neurológicos, IC derecha y FMO fueron las causas o modos de muerte temprana más frecuentes después de la implantación del DAV. La infección, la FMO y eventos neurológicos mayores fueron las principales causas de mortalidad tardía. En este análisis, el riesgo de muerte por mal funcionamiento del dispositivo fue bajo y constante en el tiempo.

Las complicaciones eran más frecuentes en aquellos dispositivos pulsátiles de primera generación ${ }^{29,33}$, por lo que se realizaron análisis de rentabilidad comparando el uso de los mismos como puente a trasplante con el tratamiento médico resultando los LVAD más caros y menos beneficiosos ${ }^{77}$. Los actuales de segunda y tercera generación fueron evaluados en comparación con el tratamiento médico en un estudio de Clarke et al..$^{78}$ en donde los outcomes fueron medidos como quality-adjusted life-year (años de vida ajustados por calidad AVAC). En este estudio se vio que proporcionan mayores beneficios a costos más altos, siendo en Gran Bretaña, un valor de $£ 20.000$ a $£ 30.000$ LVAD / AVAC por lo que concluyen que no son rentables en aquel país.

En el estudio de Ammirati et al. ${ }^{79}$, en donde se comparó en un sólo centro italiano a 213 pacientes con ICA que se trataron con LVAD o con trasplante cardíaco. Se encontró que la supervivencia a medio plazo de los pacientes con LVAD no fue estadísticamente diferente. La mortalidad perioperatoria fue similar en la cohorte general y en todos los subgrupos, aunque es importante destacar que aquellos con DAV se encontraban en peores condiciones preoperatorias.

En la era actual de los DAV de flujo continuo, la carga de eventos adversos se ha reducido, pero sigue siendo alta, ya que sólo el 30\% de los pacientes están libres de cualquier complicación importante en 1 año ${ }^{78}$. Si bien las mejoras en los dispositivos y en el seguimiento, así como la detección temprana de complicaciones, con su consecuente reducción, en la última era persisten aún como principales causas de readmisión hospitalaria el sangrado gastrointestinal debido al desarrollo de las malformaciones arteriovenosas a causa de la disminución de la presión de pulso por el flujo continuo que generan 29,80 , seguido en frecuencia por el ACV y la trombosis.

\section{ROL DE LA ECOCARDIOGRAFÍA}

Actualmente el uso de la valoración ecocardiográfica tanto preoperatoria como en el seguimiento posoperatorio de los pacientes con LVAD cobró interés al conocerse mejor tanto el funcionamiento y las posibles complicaciones que estos dispositivos pueden traer.

Es así que la Sociedad Americana de Ecocardiografía publicó el año pasado las recomendaciones para la evaluación de pacientes con LVAD basada fundamentalmente en la evaluación de los dispositivos HeartMate lly HeartWare los más frecuentemente usadas como BTT y DT ${ }^{81}$.

Ambos dispositivos comparten características en su estructura: una cánula de entrada colocada en el VI cerca del vértice, un impulsor mecánico, y una cánula de salida anastomosada a la aorta ascenden-

te (Figuras 4 y 5). La ecocardiografía permite la visualización directa de estos componentes, pero no del impulsor mecánico. El impulsor de HeartMate // y su estructura de alojamiento son implantados por debajo del diafragma, mientras que el impulsor HeartWare y su estructura de alojamiento se implantan por encima del diafragma, dentro del saco pericárdico. El posicionamiento del impulsor es el factor de diferenciación primaria en la evaluación ecocardiográfica del flujo de entrada de estos dos dispositivos ${ }^{24,39}$.

La evaluación del candidato a LVAD debe ser realizada por un ecocardiografista experimentado en ICA y en los parámetros hemodinámicos de los DAV. Generalmente un ecocardiograma transtoráciCo (ETT) es la evaluación de primera línea, tratando de buscar alteraciones anatómicas y funcionales que podrían impedir la colocación del soporte ventricular como por ejemplo, un pequeño tamaño del VI, trombos intracavitarios, aneurisma apical del VI, defectos en el septum interventricular, un VD dilatado o disfuncionante, así como también anormalidades valvulares ${ }^{81}$.

La correcta cuantificación de la FEVI es central, puesto que la determinación de una FEVI menor de 25\% es un requerimiento para ser un potencial candidato a LVAD así como también es necesaria para el cálculo de los score de riesgo. Se recomienda la cuantificación de la misma con el método tridimensional o por el bidimensional utilizando el método de Simpson, considerando la realización de contraste para mejorar la definición del endocardio y así obtener mejor precisión. Los diámetros internos del VI y el volumen de fin de diástole deben medirse para así obtener una mejor cuantificación de la disminución de la precarga del VI en posoperatorio ${ }^{81.82}$

Teniendo en cuenta el impacto de la disfunción del VD, existe un renovado interés en el uso de los parámetros ecocardiográficos para su evaluación. Signos ecocardiográficos de disfunción del VD incluyen deterioro de la función sistólica del VD y/o dilatación del VD, aumento de la presión de la aurícula derecha (comprobada por el tamaño de la vena cava inferior y su colapso), la insuficiencia tricuspídea moderada o severa ${ }^{81}$ 
Investigadores de la Cleveland Clinic utilizan medidas cuantitativas de la función del VD para evaluar el riesgo de falla del VD por ecocardiografía. En el trabajo de Grant et al. ${ }^{83}$ los pacientes que requerían un DAV derecha o soporte inotrópico prolongado (4-14 días) eran más propensos a tener reducido el strain de la pared libre del VD. Un punto de corte de $-9.6 \%$ para la máxima deformación se asoció con mayor sensibilidad y especificidad para predecir la insuficiencia del VD. En el estudio de Vivo et al. ${ }^{84}$, se vio una relación de diámetro VD/NI de $\geq 0,75$ se asoció significativamente con el outcome primario de falla ventricular derecha y el end point secundario de falla ventricular derecha a los 30 días o muerte entre los pacientes con LVAD de flujo continuo.

Clínicamente una disfunción biventricular severa perioperatoria puede incitar al equipo de IC a considerar un BiDAV en forma precoz, ya que esto puede conducir a mejores resultados que la posterior conversión de un LVAD a una asistencia biventricular tal como se vio en el estudio de Fitzpatrick et al. ${ }^{41}$ en donde el grupo de asistencia biventricular precoz tenía una supervivencia superior al alta que los pacientes en el grupo de demora a la BiVAD (51 vs. 29\%, p <0,05).

Algunos pacientes con menor disfunción del VD en la evaluación preoperatoria desarrollarán severa disfunción ventricular derecha después de la implantación del LVAD. Esta complicación, que se define por INTERMACS ${ }^{85}$ como la exigencia de un dispositivo de asistencia ventricular derecho (right ventricular assist device [RVAD]) o > 14 días consecutivos de soporte inotrópico por vía intravenosa, tiene una prevalencia estimada del 13 al 44\% y se asocia con una morbimortalidad significativa, particularmente porque el perfil hemodinámico y las características de quienes requieren un BiVAD denotan peor estado general. En el trabajo de Cleveland et al. ${ }^{86}$, el mayor requerimiento de inotrópicos, los peores parámetros de laboratorio presentaron mejores desenlaces con la colocación precoz de una BiVAD.

Dada la falta de consenso hasta el momento en relación con el valor predictivo de cualquier parámetro ecocardiográfico aislado, las guías de práctica clínica ${ }^{81}$ no recomienda utilizar un sólo parámetro para evaluar la disfunción derecha sino, utilizar tanto parámetros izquierdos y derechos para la decisión de un BiVAD.

Al momento de la colocación del DAV un ecocardiograma transesofágico (ETE) debe ser realizado en el quirófano, antes de la colocación de la misma para confirmar lo previamente analizado. Se monitoriza por ETE el momento de encender el equipo. Se buscan posibles complicaciones post implante como derrame pericárdico, mayor disfunción ventricular secundaria a disminución brusca de la carga ventricular, falla del VD, presiones de llenado inadecuadas por dimensiones demasiado pequeñas del VI que pueden deberse a falla derecha o a una velocidad excesiva de la bomba. Otra consecuencia de una velocidad elevada es la succión que puede generar el dispositivo viéndose un segmento de miocardio que ocluye parcialmente la cánula de entrada y reduce flujo de entrada de la bomba ${ }^{81}$.

Luego de implantado el dispositivo se deben realizar evaluaciones ecocardiográficas a diferentes intervalos de tiempo para evaluar la respuesta del paciente al dispositivo, siendo estas coordinadas con las visitas de rutina que se hace al equipo de IC, en donde además del examen físico de rutina se indica para valoración de todos los parámetros antedichos para la búsqueda de posibles complicaciones asociadas al DAV ${ }^{81}$.

\section{DISCUSIÓN}

Actualmente, la mayor partre de la literatura a nivel mundial se refiere a la IC como una enfermedad epidémica, por su aumento progresivo en incidencia, prevalencia, morbimortalidad y los costos que implica esto en la salud pública. En consecuencia, también aumenta el número de pacientes en ICA, (estadio D de la AHA) 1,3. En esta instancia, el manejo del paciente es complejo, llegando a la necesidad de evaluar al paciente como candidato a trasplante.

Así como se han producido cambios epidemiológicos en la IC, lo mismo ha sucedido con la población con indicación de trasplante con una desproporción donante/receptor. En este contexto surgen como estrategias posibles y aún en desarrollo: los cuidados paliativos y los DAV.

Aunque desde el primer trasplante cardíaco, se han ido perfeccionando las técnicas quirúrgicas, conociéndose mejor el manejo de los pacientes en el postoperatorio y se ha avanzado en la búsqueda de mejores tratamientos inmunosupresores, su escasez ha llevado a que los DAV vayan ganando terreno en el tratamiento de la ICA, intentando mejorar la sobrevida y la calidad de vida de los pacientes en estadios finales de la enfermedad ${ }^{7,11,12}$.

Con los DAV se genera un flujo circulatorio que sustituye parcial o totalmente la función del corazón tanto en situaciones de falla aguda o crónica severas que no responden al máximo tratamiento médico disponible. Estos han pasado por cambios y mejoras tecnológicas en búsqueda del dispositivo ideal: mayor durabilidad, menores tasas de complicaciones, costo razonable y mayor confort al paciente, según el escenario clínico de su implantación.

Por lo antedicho se clasifican en primera, segunda y tercera generación. Las diferencias entre ellos se relacionan principalmente con aspectos técnicos pero que se traducen en que los más nuevos se caracterizan por:

- Mayor durabilidad, alcanzando los 5 años. El desafío que afrontan los más recientes es superar este límite y así poder ser verdaderas terapias de destino.

- Menor tamaño y menor exposición de cables y tubuladuras, logrando mejor calidad de vida para el paciente, menores tiempos quirúrgicos para su implante y menores tasas de infecciones, a su vez mejor destete del dispositivo en aquellos en los que se puede llegar a explantar el mismo.

- Menor fricción entre sus componentes, aportando mejoría en la hemocompatibilidad, reduciendo las tasas de trombosis y permitiendo controles de RIN menos elevados.

Al conocerse mejor la hemodinamia de las asistencias y su efecto a nivel miocárdico, se empezaron a estudiar nuevas formas de aplicar la tecnología abarcando no sólo los pacientes con ICA crónica en plan de trasplante sino los excluidos del mismo, y aquellos con posibilidades de recuperar la función miocárdica ${ }^{41,43}$. Surgen así diferentes indicaciones para su utilización:

- Puente a trasplante.

- Terapia de destino.

- Puente a recuperación.

El conocimiento de estas indicaciones hace que durante la evaluación y el seguimiento del paciente el cambio de una estrategia a otra sea algo 
dinámico y no totalmente estructurado, por lo que en los últimos años se habla del puente a candidatura, según la evolución clínica y la decisión del paciente y su familia.

A la hora de pensar en las posibles indicaciones de los DAV, los médicos tratantes deben definir qué perfil hemodinámico posee el paciente y qué beneficios le esperan. Para ello en el año 2006 INTERMACS definió siete perfiles clínicos que permiten estratificar a los pacientes en estadio $D$ en función de variables hemodinámicas, grado de daño de órgano blanco, y así establecer la severidad de cada paciente y definir el implante de un DAV. Posteriormente, las diferentes sociedades cardiológicas y de trasplante han establecido grados de recomendaciones para las indicaciones de las mismas, así como contraindicaciones previamente enumeradas ${ }^{1,247,49}$.

En el séptimo INTERMACS ${ }^{29}$ los pacientes que recibieron un dispositivo alcanzaron un $\mathrm{N}$ de 15.000 lo que demuestra la rápida expansión de esta estrategia terapéutica. En forma más frecuente, así mismo un mayor número de centros no sólo los utilizan como puente a trasplante sino que a raíz de los resultados de los trabajos como el REMATCH ${ }^{35}$ la estrategia de terapia de destino constituye un porcentaje considerable y en crescendo, siendo en los últimos años la principal indicación ${ }^{29}$.

Uno de los mayores avances fue el campo de la recuperación miocárdica luego de la implantación del DAV, aunque la explantación con éxito del LVAD parece tener resultados razonables y prometedores, aún queda mucho por aprender acerca de esta estrategia. No se sabe si las características del paciente o de la etiología de la miocardiopatía predicen éxito. Además, se desconoce el momento y protocolo óptimo para el destete. Aunque los resultados de seguimiento a corto y medio plazo sugieren que la función del ventrículo izquierdo se mantiene estable, se necesita de mayor seguimiento para confirmar esta hipótesis.

Son consistentes los hallazgos a lo largo de los diferentes trabajos las diferentes indicaciones y el momento de oportuno de la colocación de los DAV. Siendo los perfiles de INTERMACS más altos los que más se benefician de una terapia con LVAD o BiVAD en cualquiera de sus indicaciones ${ }^{29,47,48}$. Lo que pone énfasis en que todos los pacientes con ICA deben ser evaluados por médicos especialistas en IC que puedan optimizar el tratamiento médico y la colocación de TRC y/o CDI, así como también establecer equipos multidisciplinarios para la toma de decisiones y el manejo no sólo del paciente sino también de sus familiares y acompañantes. Para ello es necesaria la rápida identificación de los síntomas y signos de ICA.

A pesar de todo esto, en las diferentes series la mayoría de los DAV son indicados a niveles de INTERMACS más bajos, cuando los pacientes se encuentran más deteriorados con mayor fallo hemodinámico, mayor porcentaje de depresión y mayores índices de fragilidad, con un mayor requerimiento de BiVAD, lo cual empeora los desenlaces y acrecienta los costos $2,29,47,48$

Diversas variables clínicas y biológicas deben alertar al médico tratante para la correcta derivación oportuna al equipo de IC, los cuales mediante los diferentes score de riesgo podrían facilitar la toma de decisiones y establecer las mejores estrategias de tratamiento².
Así como se fueron definiendo variables estrictamente relacionadas con la enfermedad, el mejor conocimiento de la evolución de estos pacientes y seguimientos más largos han llevado a establecer que existen factores extra cardíacos que también deben intervenir en la compleja toma de decisiones. Si bien en los últimos años se puso énfasis en el manejo de los pacientes ambulatorios, los mismos criterios y cuidados deben aplicarse a pacientes internados. Es imprescindible contar con estructuras de apoyo adecuadas, multidisciplinarias, aplicar scores de riesgos ya mencionados y evaluar, tratar y no subestimar factores psicosociales, principalmente la ansiedad y la depresión tanto en el pre- como en el posimplante, que pueden llevar a mayores complicaciones relacionadas con el dispositivo y a peores desenlaces tanto posimplante del DAV como postrasplante cardíaco ${ }^{52}$.

Uno de los grandes logros del desarrollo tecnológico en este campo fue el hecho de que los pacientes que antes estaban confinados a largos días de espera internados puedan volver al hogar reduciéndose así, el aislamiento social, la depresión y ansiedad, consiguiendo que el paciente pueda volver a insertarse socialmente, gane confianza en su cuidado con un mejor soporte emocional, $62-65,70$.

Todo esto lleva a la integración de especialistas en cuidados paliativos al cuidado de este tipo de pacientes y su familia, donde la sobrecarga del cuidador es uno de los puntos preocupantes que conlleva esta terapia. El arte de poder comunicarse con la familia, el paciente y de transmitirles todas las posibilidades de tratamiento así como el momento oportuno del switch off del dispositivo 71,72 .

A su vez, a pesar de los logros y mejoras mencionadas en los DAV y en la selección de pacientes, aún siguen siendo complejas las decisiones y su implementación en la práctica clínica. Siguen siendo frecuentes las complicaciones relacionadas con el dispositivo: hemorragia gastrointestinal, el ACV y las infecciones son las que lideran los porcentajes, lo que lleva a que el análisis de costos en algunos países los definan como no rentables, no solo por su costo sino por la estructura y el financiamiento educativo que conllevan.

\section{CONCLUSIÓN}

Ya desde la definición de los diferentes estadios de la AHA en el 2005 se plantea la posibilidad que aquellos en estadio $D$ podrían beneficiarse de un DAV. Como fue analizado, su uso fue extendido a diferentes contextos clínicos. En su evolución se ha logrado mejoría de las tecnologías y disminución de las complicaciones.

Sin embargo, los DAV persisten como una realidad con difícil aplicabilidad dado los costos y el escaso entrenamiento médico para su manejo. Persisten grandes expectativas a futuro y se espera que puedan ir ganando terreno en aquellos lugares que todavía no pueden financiarlos, generando lo que alguna vez el advenimiento del trasplante hizo: programas de salud y entrenamiento de los profesionales. 


\section{BIBLIOGRAFÍA}

1. Ponikowski P, Voors A, Anker SD, Bueno H, Cleland J, Coats A et al. 2016 ESC Guidelines for the diagnosis and treatment of acute and chronic heart failure. The Task Force for the diagnosis and treatment of acute and chronic heart failure of the European Society of Cardiology (ESC). Eur J Heart Fail 2016;18(37):891-975.

2. Trochua JN, Leprinceb P, Bielefeld-Gomezc M, Bastiend O, Beauvaise F, Gueffeta JP et al. Left ventricle assist device: When and which patients should we refer? Arch Cardiovasc Dis 2012;105(12):114-21.

3. Chaudhry SP, Stewart GC.Advanced Heart Failure: Prevalence, Natural History, and Prognosis. Heart Fail Clin 2016;12(2):323-33.

4. Yancy CW, Jessup M, Bozkurt B, Butler J, Casey DE Jr, Drazner MH, et al. 2013 ACCF/AHA guideline for the management of heart failure: a report of the American College of Cardiology Foundation/American Heart A ssociation Task Force on practice guidelines. Circulation 2013;128(16):e240-327.

5. Roger V. Epidemiology of Heart Failure. Circ Res 2013:113(6):646-659.

6. Síntesis Estadística 1:2016. Ministerio de Salud de la Nación. Dirección de Estadísticas e información de salud.

7. Marconi EH. Estadísticas vitales, información básica. Secretaria de politicas, regulacion e institutos. Dirección de estadísticas e información de salud. Ministerio de Salud de la Nación 2013;5:57.

8. Mozaffarian D, Benjamin EJ, Go A, Arnett D, Blaha MJ, Cushman M, et al. American Heart Association: 2001 Heart and Stroke Statistical Update. Circulation 2015;132(4):e29-322.

9. Edwards L Kucheryavaya A Benden C Christie J, Dipchand A Dobbels F et al. The Registry of the International Society for Heart and Lung Transplantation: Thir ty-first Official Adult Heart Transplant Report-2014; Focus Theme: Retransplantation. J Heart Lung Transplant 2014:33(10):996-1008.

10. Ho KKL, Pinsky JL, Kannel WB, Levy D.The epidemiology of heart failure: the Framingham Study. J Am Coll Cardiol 1993;22(4):6A-13A.

11. Stehlik J, Stevenson LW, Edwards LB, Crespo-Leiro MG, Delgado JF, Dorent $R$, et al. Organ Allocation Around the World: Insights From the ISHLT International Registry for Heart and Lung Transplantation. J Heart Lung Transplant 2014:33(10):975-84.

12. Gómez Bueno M, Segovia Cubero J, Alonso-Pulpón Rivera L. Asistencia mecánica circulatoria y trasplante cardiaco. Indicaciones y situación en España. Rev Esp Cardiol 2006:6(6):82F-94F

13. Buggey J, Mentz RJ, Galanos AN. End-of-life Heart Failure Care in the United States. Heart Fail Clin 2015;11(4):615-23

14. Van Riet EE, Hoes AW, Wagenaar KP, Limburg A, Landman MA, Rutten FH. EPidemiology of heart failure: the prevalence of heart failure and ventricular dysfunction in older adults over time. A systematic review. Eur J Heart Fail 2016;18(19):242-52.

15. Levy D, Kenchalah S, Larson MG, Benjamin, Kupka. Long term trends in the incidence and survival with heart failure. N Engl J Med 2002;347(18):1397-402.

16. Helman DN, Rose EA. History of Mechanical Circulatory Support. Prog CardiovasC Dis 2000;43(1):1-4

17. Tang DG1, Oyer PE, Mallidi HR. Ventricular Assist Devices: History, Patient Selec tion, and Timing of Therapy. J Cardiovasc Trans/ Res 2009:2(2):159-67.

18. Miller MA, Ulisney K, Baldwin JT. INTERMACS (Interagency Registry for Mechani cally Assisted Circulatory Support): a new paradigm for translating registry data into clinical practice. J Am Coll Cardiol 2010;56(9):738-40.

19. Stevenson L, Pagani F, Young JB, Jessup M, Miller L, Kormos RL et al. INTERMACS Profiles of Advanced Heart Failure: The Current Picture. J Heart Lung Transplant 2009:28(6):535-41.

20. Barge Caballeroa E, Paniagua M, Marzoa Rivasa M, Campo Pereza R. Utilidad de la escala INTERMACS para estratificar el pronóstico tras el trasplante cardiaco urgente. Rev Esp Cardiol 2011;64(3):193-200.

21. Alba AC, Rao V, Ivanov J, Ross HJ, Delgado DH. Usefulness of the INTERMACS scale to predict outcomes after mechanical assist device implantation. J Heart Lung Transplant 2009:28(8):827-33.

22. Carpentier A, Latrémouille C, Cholley B, Smadja DM, Roussel JC, Boissier J et al. First clinical use of a bioprosthetic total artificial heart: report of two cases. Lancet 2015:386(10003):1556-63.

23. Stewart GC, Givertz MM.Mechanical circulatory support for advanced heart failure: patients and technology in evolution. Circulation 2012;125(10):1304-15.

24. Kantrowitz A, Tjonneland S, Freed PS, Phillips SJ, Butner AN, Sherman JL Jr. Initial clinical experience with intraaortic balloon pumping in cardiogenic shock. JAMA 1968;203(2):113-8

25. Mussivand T. Mechanical circulatory devices for the treatment of Heart Failure. $J$ Card Surg 1999;14(3):218-28

26. Delgado DH, Rao V, Ross HJ, Verma S, Smedira NG. Mechanical Circulatory Assistance State of Art. Circulation 2002;106(8):2046-50.

27. Kutty RS, Parameshwar J, Lewis $C$, Catarino PA, Sudarshan $C D$, Jenkins DP, et al. Use of centrifugal left ventricular assist device as a bridge to candidacy in severe heart failure with secondary pulmonary hypertension. Eur I Cardiothorac Surg 2013:43(6):1237-42.
28. Kar B, Gregoric ID, Basra SS, Idelchik GM, Loyalka P. The percutaneous ven tricular assist device in severe refractory cardiogenic shock. J Am Coll Cardio 2011;57(6):688-96

29. Kirklin JK, Naftel DC, Pagani FD, Kormos RL, Stevenson LW, Blume ED, et al. Seventh INTERMACS annual report: 15,000 patients and counting. J Heart Lung Transplant 2015;34(12):1495-504

30. Rose EA, Gelijns AC, Moskowitz AJ, Heitjan DF, Stevenson LW, Dembitsky W et al Long-term use of a left ventricular assist device for end-stage heart failure. Randomized Evaluation of Mechanical Assistance for the Treatment of Congestive Heart Failure (REMATCH). N Engl J Med 2001;345(20):1435.

31. Nguyen $D Q$, Thourani VH. Third-generation continuous flow left ventricular assis devices. Innovations (Phila) 2010;5(4):250-8.

32. Ashleigh Xie, Kevin Phan, Tristan D. Yan Durability of continuous-flow left ventric ular assist devices: a systematic review Ann Cardiothorac Surg 2014:3(6):547-556.

33. Netuka I, Beyersdorf F, Garbade J, Krabatsch T, Marasco S, Morshuis M, et al HeartMate 3 fully magnetically levitated LVAD for the treatment of advanced heart failure: results from the CE Mark Trial. Presented at the 19th Annual Meeting of the Heart Failure Society of America (HFSA) 2015;12(1):23.

34. Burke DJ, Burke E, Parsaie F, Poirier V, Butler K, Thomas D et al. The Heartmate II: design and development of a fully sealed axial flow left ventricular assist system. Artif Organs 2001;25(2):380-5

35. Frazier OH, Myers TJ, Westaby S, Gregoric ID. Clinical experience with an im plantable, intracardiac, continuous flow circulatory support device: physiologic implications and their relationship to patient selection. Ann Thorac Surg 2004; $77(1): 133-42$

36. Sabashnikov A Mohite PN Simon AR Popov AF HeartWare miniaturized intrapericardial ventricular assist device: advantages and adverse events in comparison to contemporary devices. Expert Rev Med Devices 2013;10(4):441-52.

37. Capdeville M. Smedira NG. Advances and future directions for mechanical circulatory support. Anesthesiol Clin 2013;31(2):321-53.

38. Hanke JS, Rojas SV, Avsar M, Bara C, Ismail I, Haverich A, et al. HeartWare left ventricular assist device for the treatment of advanced heart failure. Future Cardio 2016;12(1):17-26.

39. Milano CA, Pagani FD, Tatooles AJ, Mokadam NA, Miller J, Wozniak TC, et al. Temporal Changes in Adverse Events in the ENDURANCE Trial. J Heart Lung Transplant 2013;32(2):10

40. Rihal CS, Naidu SS, Givertz MM, Szeto W, Burke J, Kapur N, Srihari SN, et al. 2015 SCAI/ACC/HFSA/STS Clinical Expert Consensus Statement on the Use of Percutaneous Mechanical Circulatory Support Devices in Cardiovascular Care: Endorsed by the American Heart Association, the Cardiological Society of India, and Sociedad Latino Americana de Cardiología Intervencionista; Affirmation of Value by the Canadian Association of Interventional Cardiology-Association Canadienne de Cardiologie dintervention. J Am Coll Cardiol. 2015:65(19):e7-e26.

41. Fitzpatrick JR, Frederick JR, Hiesinger W, Hsu VM, McCormick RC, Kozin ED, et al. Early planned institution of biventricular mechanical circulatory support results in improved outcomes compared with delayed conversion of a left ventricular assist device to a biventricular assist device. J Thorac Cardiovasc Surg 2009;137(4):971-7.

42. Rogers JG, Butler J, Lansman SL, Gass A, Portner PM, Pasque MK, Pierson RN. Chronic mechanical circulatory support for inotrope-dependent heart failure patients who are not transplant candidates: results of the INTrEPID Trial. J Am Coll Cardiol. 2007:50(8):741-7.

43. Birks EJ, George RS, Hedger M, Bahrami T, Wilton P, Bowles CT, et al. Reversal of severe heart failure with a continuous-flow left ventricular assist device and pharmacological therapy: a prospective study. Circulation 2011:123(4):381-90.

44. Akil A, Fischer D, Holthaus AJ, Martens S, Scherer M, Sindermann JR. Left Ventricular Assist Devices as Bridge to Cardiac Recovery in Nonischemic Heart Failure: Keeping Weaning from the Device in Mind. Thorac Cardiovasc Surg. 2016;64(6):483-6.

45. Birks EJ, Tansley PD, Hardy J, George RS, Bowles CT, Burke M et al. Left ventricular assist device and drug therapy for the reversal of heart failure. N Engl I Med 2006;355(18):1873-84.

46. Deng MC, Edwards LB, Hertz MI, Rowe AW, Keck BM, Kormos $R$, et al. Mechan cal Circulatory Support Device Database of the International Society for Heart and Lung Transplantation: second annual report-2004. J Heart Lung Transplant. 2004;23(9):1027-34.

47. Miller LW, Guglin M. Patient selection for ventricular assist devices: a moving target. J Am Coll Cardiol. 2013;61(12):1209-21.

48. Jorde UP, Kushwaha SS, Tatooles AJ, Naka Y, Bhat G, Long JW et al. Results of the destination therapy post food and drug administration approval study with a continuous flow left ventricular assist device: a prospective study using the INTERMACS registry (Interagency Registry for Mechanically Assisted Circulatory Support). J Am Coll Cardiol 2014;63(17):1751-1757. 
49. Estep JD, Starling RC, Horstmanshof DA, Milano CA, Selzman CH, Shah KB, et al. Risk assessment and comparative effectiveness of left ventricular assist device and medical management in ambulatory heart failure patients: results from the ROADMAP study. J Am Coll Cardiol 2015;66(16):1747-1761.

50. Metra M, Ponikowski P, Dickstein K, McMurray JJ, Gavazzi A, Bergh CH, et al. Advanced chronic heart failure: a position statement from the Study Group on Advanced Heart Failure of the Heart Failure Association of the European Society of Cardiology. Eur J Heart Fail 2007;9(6-7):684-94.

51. Adamson RM, Stahovich M, Chillcott S, Baradarian S, Chammas J, Jaski B et al. Clinical strategies and outcomes in advanced heart failure patients older than 70 years of age receiving the HeartMate II left ventricular assist device: a community hospital experience. J Am Coll Cardiol 2011;57(25):2487-95.

52. Feldman D, Pamboukian SV, Teuteberg JJ, Birks E, Lietz K, Moore SA et al. International Society for Heart and Lung Transplantation. The 2013 International Society for Heart and Lung Transplantation Guidelines for mechanical circulatory support: executive summary. J Heart Lung Transplant 2013:32(2):157-87.

53. Dunlay SM, Park SJ, Joyce LD, Daly RC, Stulak JM, MCNallan SM et al. Frailty and outcomes after implantation of left ventricular assist device as destination therapy. J Heart Lung Transplant 2014;33(4):359-65.

54. Mancini D, Lietz K. Selection of Cardiac Transplantation Candidates in 2010. Circulation 2010;122(2):173-183.

55. Peura JL, Colvin-Adams M, Francis GS, Grady KL, Hoffman TM, Jessup M, Recommendations for the use of mechanical circulatory support: device strategies and patient selection: a scientific statement from the American Heart Association. Circulation 2012:126(22):2648-67.

56. Levy WC, Mozaffarian D, Linker DT, Sutradhar SC, Anker SD, Cropp AB et al. The Seattle Heart Failure Model: prediction of survival in heart failure. Circulation 2006:113(11):1424-33.

57. Ketchum ES, Moorman AJ, Fishbein DP, Mokadam NA, Verrier ED, Aldea GS et al. Predictive value of the Seattle Heart Failure Model in patients undergoing left ventricular assist device placement. J Heart Lung Transplant 2010;29(9):1021-5.

58. Teuteberg JJ, Ewald GA, Adamson RM, Lietz K, Miller LW, Tatooles AJ et al. Risk assessment for continuous flow left ventricular assist devices: does the destina tion therapy risk score work? An analysis of over 1,000 patients.J Am Coll Cardiol 2012;60(1):44-51

59. Kamath PS, Wiesner RH, Malinchoc M, Kremers W, Therneau TM, Kosberg CL, et al. A model to predict survival in patients with end-stage liver disease. Hepatology 2001;33(2):464-70.

60. Matthews JC, Pagani FD, Haft JW, Koelling TM, Naftel DC, Aaronson KD. Model for end-stage liver disease score predicts left ventricular assist device operative transfusion requirements, morbidity, and mortality. Circulation 2010;121(2):214-20.

61. Yang JA, Kato TS, Shulman BP, Takayama H, Farr M, Jorde UP, et al. Liver dysfunction as a predictor of outcomes in patients with advanced heart failure requiring ventricular assist device support: Use of the Model of End-stage Liver Disease (MELD) and MELD excluding INR (MELD-XI) scoring system. J Heart Lung Transplant 2012;31(6):601-10.

62. Cowger J, Sundareswaran K, Rogers JG. Predicting survival in patients receiving continuous flow left ventricular assist devices: the HeartMate II risk score. J Am Coll Cardiol 2013;61(3):313-21.

63. Matthews JC, Koelling TM, Pagani FD, Aaronson KD. The right ventricular failure risk score a pre operative tool for assessing the risk of right ventricular failure in left ventricular assist device candidates. J Am Coll Cardiol 2008;51 (22):2163-72.

64. Spaderna H, Mendell NR, Zahn D, Wang Y, Kahn J, Smits JM et al. Social isolation and depression predict 12-month outcomes in the "waiting for a new heart study." J Heart Lung Transplant 2010;29(12):247-54

65. Spaderna H, Weidner G, Koch KC, Kaczmarek I, Wagner FM, Smits JM. Medical and psychosocial predictors of mechanical circulatory support device implantation and competing outcomes in the Waiting for a New Heart Study. J Heart Lung Transplant 2012;31(12):16-26.

66. Ye C, Zhuang Y, Zhang Y, Lin Y, Ji J, Chen H. Anxiety, depression, and associated factors among inpatients waiting for heart transplantation. Shanghai Arch Psychiatry 2013;25(21):165-73.

67. Reynard AK, Butler RS, MCKee MG, Starling RC, Gorodeski EZ. Frequency of depression and anxiety before and after insertion of a continuous flow left ventricular assist device. Am J Cardiol 2014;114(3):433-40.
68. Bruce CR, Delgado E, Kostick K, Grogan S, Ashrith G, Trachtenberg B, et al. Ventric ular assist devices: a review of psychosocial risk factors and their impact on outcomes.J Card Fail 2014;20(12):996-1003

69. Gordon RJ, Weinberg AD, Pagani FD, Slaughter MS, Pappas PS, Naka Y et al. Prospective, multicenter study of ventricular assist device infections. Circulation 2013;127(6):691-702.

70. Snipelisky D, Stulak JM, Schettle SD, Sharma S, Kushwaha SS, Dunlay SM. Psychosocial characteristics and outcomes in patients with left ventricular assist device implanted as destination therapy. Am Heart J 2015;170(5):887-94.

71. Grady KL, Magasi S, Hahn EA, Buono S, McGee EC Jr, Yancy C. Health-related quality of life in mechanical circulatory support: Development of a new conceptual model and items for self-administration. J Heart Lung Transplant 2015;34(10):1292-304.

72. Emin A, Rogers CA, Banner NR. Quality of life of advanced chronic heart failure: medical care, mechanical circulatory support and transplantation. Eur J Cardiothorac Surg 2016;50(2):269-73.

73. Luo N, Rogers JG, Dodson GC, Patel CB, Galanos AN, Milano CA, O'Connor CM, Mentz RJ. Usefulness of Palliative Care to Complement the Management of Pa tients on Left Ventricular Assist Devices. Am J Cardiol 2016:1:118(5):733-8.

74. Swetz KM, Ottenberg AL, Freeman MR, Mueller PS. Palliative care and end-of-life issues in patients treated with left ventricular assist devices as destination therapy Curr Heart Fail Rep 2011;8(3):212-8.

75. Kitko LA, Hupcey JE, Gilchrist JH, Boehmer JP. Caring for a spouse with end-stage heart failure through implantation of a left ventricular assist device as destination therapy. Heart Lung 2013:42(3):195-201.

76. Sutcliffe P, Connock M, Pulikottil-Jacob R, Kandala NB, Suri G, Gurung T, et al. Clinical effectiveness and cost-effectiveness of second-and third-generation left ventricular assist devices as either bridge to transplant or alternative to transplant for adults eligible for heart transplantation: systematic review and cost-effectivenes model. Health Technol Assess 2013;17(53):1-499.

77. Clegg AJ, Scott DA, Loveman E, Colquitt J, Hutchinson J, Royle P, et al. The clinical and cost-effectiveness of left ventricular assist devices for end-stage heart failure: a systematic review and economic evaluation. Health Technol Assess 2005;9(45):1-132.

78. Clarke A, Pulikottil-Jacob R, Connock M, Suri G, Kandala NB, Maheswaran H, et al. Cost- effectiveness of left ventricular assist devices (LVADs) for patients with advanced heart failure: analysis of the British NHS bridge to transplant (BTT) program. Int J Cardiol 2014;171(3):338-45.

79. Ammirati E, Oliva FG, Colombo T, Russo CF, Cipriani MG, Garascia A et al. Midterm survival after continuous-flow left ventricular assist device versus heart transplantation. Heart Vessels 2016;31(5):722-33.

80. Patel CB, Cowger JA, Zuckermann A. A contemporary review of mechanical circulatory support. J Heart Lung Transplant 2014;33(7):667-74.

81. Stainback RF, Estep JD, Agler DA, Birks EJ, Bremer M, Hung J et al. Echocardiography in the Management of Patients with Left Ventricular Assist Devices: Recom mendations from the American Society of Echocardiography. J Am Soc Echocardiogr. 2015;28(8):853-909

82. Lang RM, Badano LP, Mor-Avi V, Afilalo J, Armstrong A, Ernande L, et al. Recommendations for cardiac chamber quantification by echocardiography in adults: an update from the american society of echocardiography and the European as sociation of cardiovascular imaging. J Am Soc Echocardiogr 2015;28(1):1-39.

83. Grant $A D$, Smedira NG, Starling $R C$, Marwick TH. Independent and incremental role of quantitative right ventricular evaluation for the prediction of right ventricular failure after left ventricular assist device implantation. J Am Coll Cardiol 2012;60(6):521-8.

84. Vivo RP, Cordero-Reyes AM, Qamar U, Garikipati S, Trevino AR, Aldeiri M et al. Increased right-to-left ventricle diameter ratio is a strong predictor of right ventricular failure after left ventricular assist device. J Heart Lung Transplant 2013;32(8):792-9.

85. Kirklin JK, Naftel DC, Kormos RL, Stevenson LW, Pagani FD, Miller MA, et al. Fifth INTERMACS annual report: risk factor analysis from more than 6,000 mechanical circulatory support patients. J Heart Lung Transplant 2013;32(2):141-56.

86. Cleveland JC Jr, Naftel DC, Reece TB, Murray M, Antaki J, Pagani FD, Kirklin JK. Survival after biventricular assist device implantation: an analysis of the Interagency Registry for Mechanically Assisted Circulatory Support database. J Heart Lung Transplant 2011;30(8):862-9. 